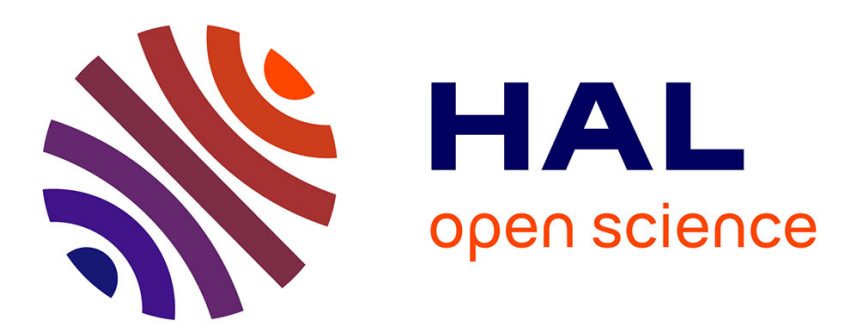

\title{
Parures et territoires à la fin de l'âge du Bronze Les bracelets du groupe d'Orgon dans le Sud-Est de la France
}

Thibault Lachenal

\section{- To cite this version:}

Thibault Lachenal. Parures et territoires à la fin de l'âge du Bronze Les bracelets du groupe d'Orgon dans le Sud-Est de la France. Bulletin de la Société préhistorique française, 2017, 114 (3), pp.553-572. halshs-01837088

\section{HAL Id: halshs-01837088 \\ https://shs.hal.science/halshs-01837088}

Submitted on 4 Feb 2020

HAL is a multi-disciplinary open access archive for the deposit and dissemination of scientific research documents, whether they are published or not. The documents may come from teaching and research institutions in France or abroad, or from public or private research centers.
L'archive ouverte pluridisciplinaire HAL, est destinée au dépôt et à la diffusion de documents scientifiques de niveau recherche, publiés ou non, émanant des établissements d'enseignement et de recherche français ou étrangers, des laboratoires publics ou privés. 


\title{
Parures et territoires à la fin de l'âge du Bronze
}

\section{Les bracelets du groupe d'Orgon dans le Sud-Est de la France}

\author{
Thibault LACHENAL
}

Résumé : Cette étude s'intéresse à une variété de parure annulaire dont l'originalité se devait d'être soulignée, qu'il est proposé de nommer bracelets du groupe d'Orgon en référence au dépôt de Notre-Dame de Beauregard, dans cette localité des Bouches-du-Rhône, qui en a livré le plus grand nombre. Ce groupe est illustré par quarante objets provenant de douze sites répartis dans quatre départements du Sud-Est de la France. Il correspond à des bracelets ouverts, de tige mince à section en D aplati dont les extrémités sont ornées de bourrelets transversaux. Les contextes de découvertes de ces objets, de nature variée (habitats, dépôts et nécropoles) convergent vers une datation de leur production au Bronze final $3 b$ et éventuellement lors de la phase de transition entre l'âge du Bronze et l'âge du Fer, aux IX ${ }^{\mathrm{e}}$ et VIII ${ }^{\mathrm{e}} \mathrm{s}$. av. J.-C. Ces parures sont munies de décors couvrant la totalité du jonc et présentant une certaine variabilité en fonction de la technique utilisée et des motifs représentés. Leur combinaison permet de définir 3 types qui s'excluent géographiquement, la majorité des exemplaires de chacun d'entre eux se concentrant dans un cercle ne dépassant pas les $50 \mathrm{~km}$ de diamètre. Ces différences peuvent signaler l'existence de trois ateliers spécialisés possédant chacun un type d'ornementation spécifique. Plusieurs indices, comme un moule, témoignent en effet d'une production locale de ces objets. Les bracelets de même type provenant du dépôt de Notre-Dame de Beauregard et de la nécropole des Lauzières à Lourmarin dans le Vaucluse présentent par ailleurs des signatures élémentaires semblables, qui laissent penser qu'ils proviennent d'un même atelier. Une autre hypothèse expliquant la production de ces types est que ces décors furent les supports d'expressions identitaires et que leur zone de répartition principale corresponde à un territoire communautaire. Une bonne correspondance peut en effet être établie entre la répartition de ces objets et les styles céramiques du bassin inférieur du Rhône à l'extrême fin de l'âge du Bronze. Localisées à distance des ressources en cuivre des Alpes et du Sud du Massif central, ces sociétés ne sont néanmoins pas dépourvues d'une production métallurgique propre, dont les bracelets du groupe d'Orgon constituent les témoins les plus directs.

Mots-clefs : Bronze final, Sud-Est de la France, bracelets, parures annulaires, décors, typologie, territoires, ateliers.

Abstract: This paper focuses on a variety of annular ornament whose originality should be emphasized, and which we propose to call Orgon group bracelets in reference to a hoard discovered in Notre-Dame de Beauregard (Bouchesdu-Rhône) which contained the majority of the known objects. This group is illustrated by fourty objects, to which must be added a possible casting mould, from twelve sites spread over four departments of southeastern France: Bouchesdu-Rhône, Alpes-de-Haute-Provence, Vaucluse and Ardèche (see table 1). It corresponds to an open bracelet provided with a thin stem with a flattened D-section the ends of which are decorated with transverse ribs. These bracelets come from varied contexts (human settlements, hoards and tombs; fig. 12) and can be dated to the Late Bronze Age 3b, or possibly to the transition phase between the Bronze Age and the Iron Age (i.e. between the 9th and 8th centuries BC). These ornaments probably are objects of everyday life, found in the form of fragments on settlements, but which can also be parts of much more ostentatious ornaments, as illustrated by the rich costume of the hoard of the Déroc cave. The tombs discoveries indicate that they may have been worn either singly or in series. The tomb of the Lauzières and the two hoards clearly illustrate this, with increasing lengths allowing the shape of the forearm to be matched (fig. 13). They are provided with decorations covering the whole bangle and presenting a certain variability according to the technique used and the patterns depicted. The combination of the latter makes it possible to define 3 types: the Lauzières type corresponding to the specimens supporting only a relief decoration, the Notre-Dame de Beauregard type type characterized by engraved decors of lines, crosses and triangles, finally the Déroc type, also determined by the presence of engraved ornamentations, which combines the cruciform decorations with ovoid patterns (fig. 10). These decorative types find some correspondence with the morphology of the objects, considering their maximum length and width (fig. 11). These three different types appear to be not only located in distinct geographical areas, but also concentrated in circles which do not exceed $50 \mathrm{~km}$ in diameter (fig. 14). These differences may indicate the existence of three specialized workshops, each with a specific type of ornamentation. Several indications testify to a local production of these objects, which could proceed from the evolution of the Pourrières type, dating back to the Late Bronze Age 2b-3a. First of all, we can evoke the presence of a casting mould (fig. 3, no. 3) coming from the tumulus of the Moullard sud in Lambesc (Bouches-du-Rhône), which was transformed in a second stage for the production of socketed axes. Secondly 
we can notice that the type of bracelets discovered in the hoard of Notre-Dame de Beauregard and in the Lauzières necropolis have similar elementary signatures, which suggest that they originated from the same workshop (fig. 15). They are moreover similar to those of other objects coming from the Southern Alps, where this group of bracelet is unknown (fig. 16). Its creation could therefore correspond to a desire to produce less massive, less metal-consuming annular ornaments in an area located further from copper resources. Another hypothesis explaining the production of these types is that these decorations express identity and that their main geographical distribution corresponds to the territory of a human community. A good correspondence can be established between the distribution of these objects and the ceramic styles of the lower Rhone basin at the end of the Bronze Age. However, regardless of the fact they are located quite far from the copper resources of the Alps and the south of the Massif Central, these societies have their own metallurgical production, of which the Orgon group bracelets constitute the most direct witnesses.

Keywords: Late Bronze Age, Southern France, bracelets, annular ornaments, geometric design, typology, territories, workshops.

\section{À André Müller}

$\mathrm{C}$ ETTE ÉTUDE s'inscrit dans la continuité des travaux initiés par A. Müller sur le mobilier métallique de la nécropole des Lauzières à Lourmarin dans le Vaucluse et du dépôt de Notre-Dame de Beauregard à Orgon dans les Bouches-du-Rhône (Müller, 2004). Elle s'intéresse plus particulièrement à une variété de parure annulaire présente dans ces deux ensembles, dont l'originalité dans le Sud-Est de la France se devait d'être soulignée. Cette dernière variété de parures se retrouve par ailleurs dans plusieurs sites d'habitat de la fin de l'âge du Bronze de basse Provence occidentale ayant fait l'objet d'une étude récente de leur mobilier céramique (Lachenal, 2011). L'objectif de cet article est d'en établir les caractéristiques typologiques et d'analyser leur répartition géographique, afin de voir en quoi elles peuvent traduire des zones de productions ou des réseaux d'échanges éclairant un aspect des territoires des sociétés protohistoriques du Sud-Est de la France.

\section{LES BRACELETS DU GROUPE D'ORGON : DÉFINITION}

1 s'agit de parures annulaires identifiables comme des bracelets. La forme en est simple, ouverte, la tige mince à section en $\mathrm{D}$ aplati et les extrémités, qui ne possèdent pas de tampons à proprement parler, sont ornées de deux ou trois bourrelets transversaux. La tige est ornée de motifs géométriques gravés de croix de saint André, de chevrons imbriqués, de triangles hachurés, d'oves, ou de cannelures obliques alternées obtenues au moment de la coulée. Cette dernière devait s'effectuer dans un moule à valve unique, le ruban ainsi obtenu étant cintré par la suite. Le choix de la dénomination de ce groupe de bracelet vient du fait que la plus grande concentration connue à ce jour se retrouve dans le dépôt de Notre-Dame de Beauregard à Orgon. Il se distingue du type de Vénat qui présente des caractères communs, comme les extrémités à bourrelets multiples, mais dont la tige est plus grêle, de section circulaire, plano-convexe ou lenticulaire (Coffyn et al. 1981; Eluère et Gomez 1990, nº 2311).

\section{INVENTAIRE DES DÉCOUVERTES}

$\mathrm{C}$ e groupe de bracelet est illustré par quarante objets provenant de douze sites, auxquels il faut ajouter un possible moule, répartis dans quatre départements du Sud-Est de la France : les Bouches-du-Rhône, les Alpesde-Haute-Provence, le Vaucluse et l'Ardèche (table 1).

\section{Alpes-de-Haute-Provence}

\section{La grotte Murée, Montagnac-Montpezat}

La grotte Murée (ou grotte A) est une vaste galerie d'une soixantaine de mètres est localisée sur la rive droite des moyennes gorges du Verdon, dans le canyon de Baudinard. Elle aurait fait l'objet d'un petit sondage vers 1951-1952 par M. Charpenel, puis a été fouillée de 1958 à 1963, à l'occasion du programme de sauvetage archéologique des gorges du Verdon. La séquence holocène, qui enregistrait des occupations s'échelonnant du Néolithique moyen à l'âge du Bronze final, a été explorée par C. Lagrand et J. Courtin. Le fragment de bracelet assimilé au groupe d'Orgon (fig. 1, $\mathrm{n}^{\circ} 1$ ) provient de la couche 4B, attribuée au « Bronze final II » (Lagrand, 1968 , p. 276). Néanmoins, l'analyse des remontages céramiques et l'étude typo-chronologique du mobilier ont montré que la stratigraphie de la grotte avait subi d'importants remaniements et que cette couche rassemble du mobilier hétérogène s'échelonnant du Bronze moyen $1 \mathrm{au}$ Bronze final $3 b^{(1)}$ (Lachenal, 2009 et 2010).

Le fragment en question correspond à une moitié de bracelet, replié à l'inverse de son cintre originel. Il est orné d'un décor plastique obtenu par moulage constitué de groupes de cannelures obliques alternées.

\section{Bouches-du-Rhône}

\section{Le domaine de l'Étoile ou Col Saint-Anne, Simiane-Col-} longue

Ce site fut découvert en 1966 par H. Puech, à la suite de l'aménagement d'un chemin carrossable destiné à faciliter la desserte d'une vigie, assurant la prévention des incendies 


\begin{tabular}{|c|c|c|c|c|c|c|c|c|c|c|}
\hline \multirow[b]{2}{*}{$\mathrm{N}^{\circ}$} & \multirow[b]{2}{*}{ Département } & \multirow[b]{2}{*}{ Commune } & \multirow[b]{2}{*}{ Site } & \multicolumn{4}{|c|}{ Type } & \multirow[b]{2}{*}{ Moule } & \multirow[b]{2}{*}{ Contexte } & \multirow[b]{2}{*}{ Datation } \\
\hline & & & & 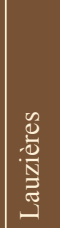 & 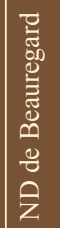 & 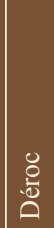 & 恋 & & & \\
\hline 1 & Alpes-de-H $\mathrm{H}^{\mathrm{te}}-$ Prov. & Montagnac-Montpezat & Grotte Murée & 1 & & & & & habitat & \\
\hline 2 & Bouches-du-Rhône & Simiane-Collongue & Domaine de l'Étoile & 2 & & & & & habitat & $\mathrm{BF} 3 \mathrm{~b}$ \\
\hline 3 & Bouches-du-Rhône & Bouc-Bel-Air & Baou-Roux & 1 & & & & & habitat & BF $3 b$ \\
\hline 4 & Bouches-du-Rhône & Berre-l'Etang & Bruny & 1 & & & & & indét. & \\
\hline 5 & Bouches-du-Rhône & Lambesc & Tumulus du Moullard & 1 & & & & 1 & funéraire & Transition Bronze/Fer \\
\hline 6 & Bouches-du-Rhône & Saint-Rémy-de-Provence & Romanin & & & & 1 & & indét. & \\
\hline 7 & Bouches-du-Rhône & Orgon & $\begin{array}{l}\text { Notre-Dame } \\
\text { de Beauregard }\end{array}$ & & 10 & & & & dépôt & BF $3 b$ \\
\hline 8 & Vaucluse & Lourmarin & Lauzières & 7 & 2 & & & & funéraire & BF $3 b$ \\
\hline 9 & Vaucluse & Buoux & Grotte du Passage & & 6 & & & & funéraire & \\
\hline 10 & Ardèche & Saint-Remèze & $\begin{array}{l}\text { Tumulus } \\
\text { de Beauregard }\end{array}$ & & 2 & 6 & & & funéraire & BF $3 b$ ou $1^{\text {er }}$ âge du Fer? \\
\hline 11 & Ardèche & Vallon-Pont-d'Arc & Grotte du Déroc & & & 4 & & & dépôt & BF $3 b$ \\
\hline 12 & Ardèche & Bérias-et-Casteljau & Casteljau & & & 2 & & & indét. & \\
\hline 13 & Vaucluse? & Orange? & & 1 & & & & & indét. & \\
\hline
\end{tabular}

Tabl. 1 - Inventaire des bracelets du groupe d'Orgon et de ses types dans le Sud-Est de la France.

Table 1 - Inventory of the bracelets of Orgon groupe and its types of southeastern France.
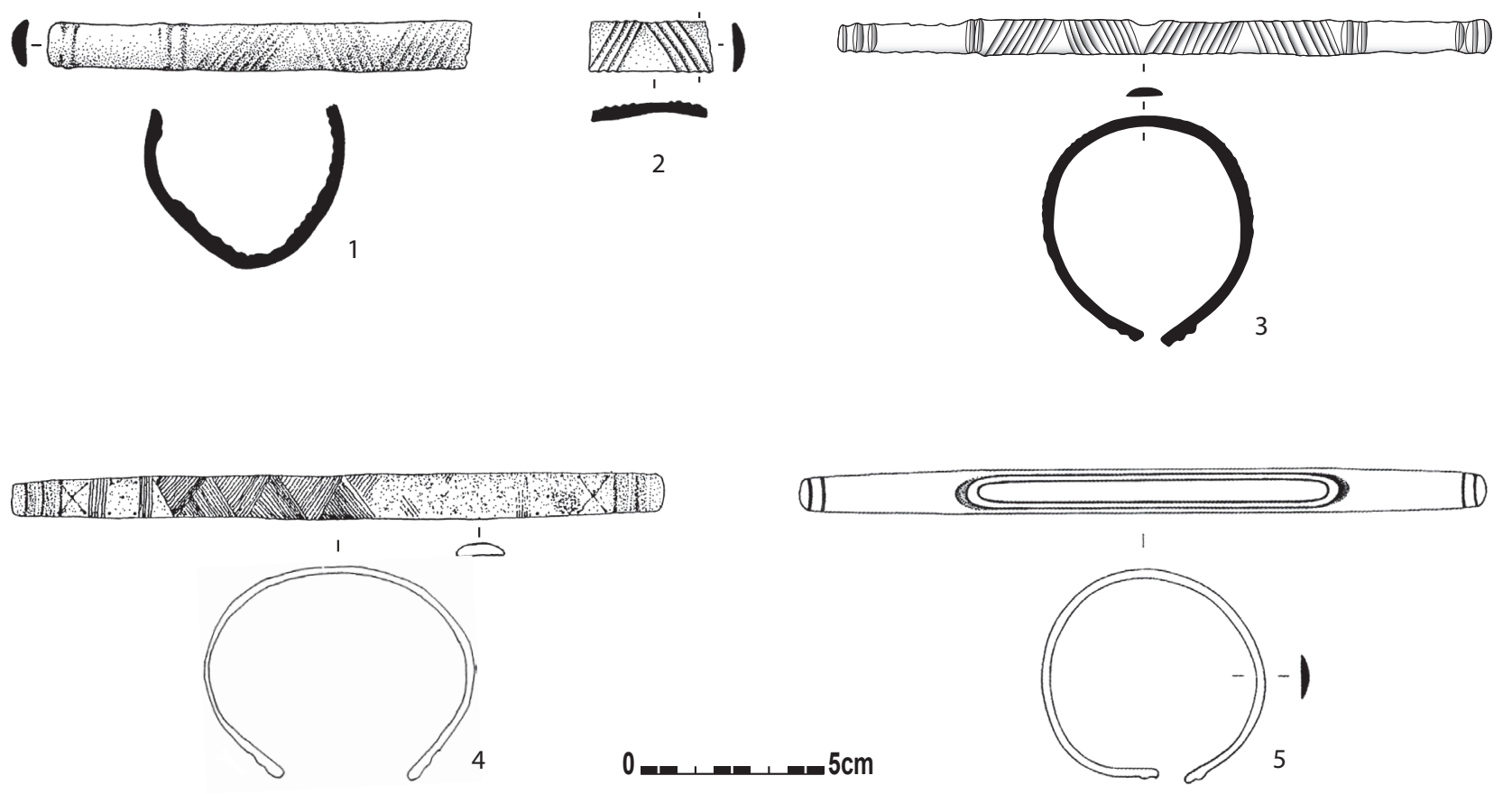

Fig. 1 - Bracelets du groupe d'Orgon. 1 : la grotte Murée, Montagnac-Montpezat, Alpes-de-Haute-Provence (d'après Lagrand, 1968); 2 : Le Baou-Roux, Bouc-Bel-Air, Bouches-du-Rhône (d'après Boissinot, 1993); 3 : " Orange, Vaucluse », collection Vallentin du Cheylard (dessin T. Lachenal); 4 : la grotte du Passage, Buoux, Vaucluse (d'après Bonnetain, 1995); 5 : Bérias-et-Casteljau, Ardèche (d'après Campolo et Garcia, 2004).

Fig. 1 - Bracelets of Orgon group. 1: Grotte Murée, Montagnac-Montpezat, Alpes-de-Haute-Provence (after Lagrand, 1968); 2: Baou-Roux, Bouc-Bel-Air, Bouches-du-Rhône (after Boissinot, 1993); 3: "Orange, Vaucluse ", from the Vallentin du Cheylard collection (drawing T. Lachenal); 4: Grotte du Passage, Buoux, Vaucluse (after Bonnetain, 1995); 5: Bérias-et-Casteljau, Ardèche (after Campolo and Garcia, 2004). 

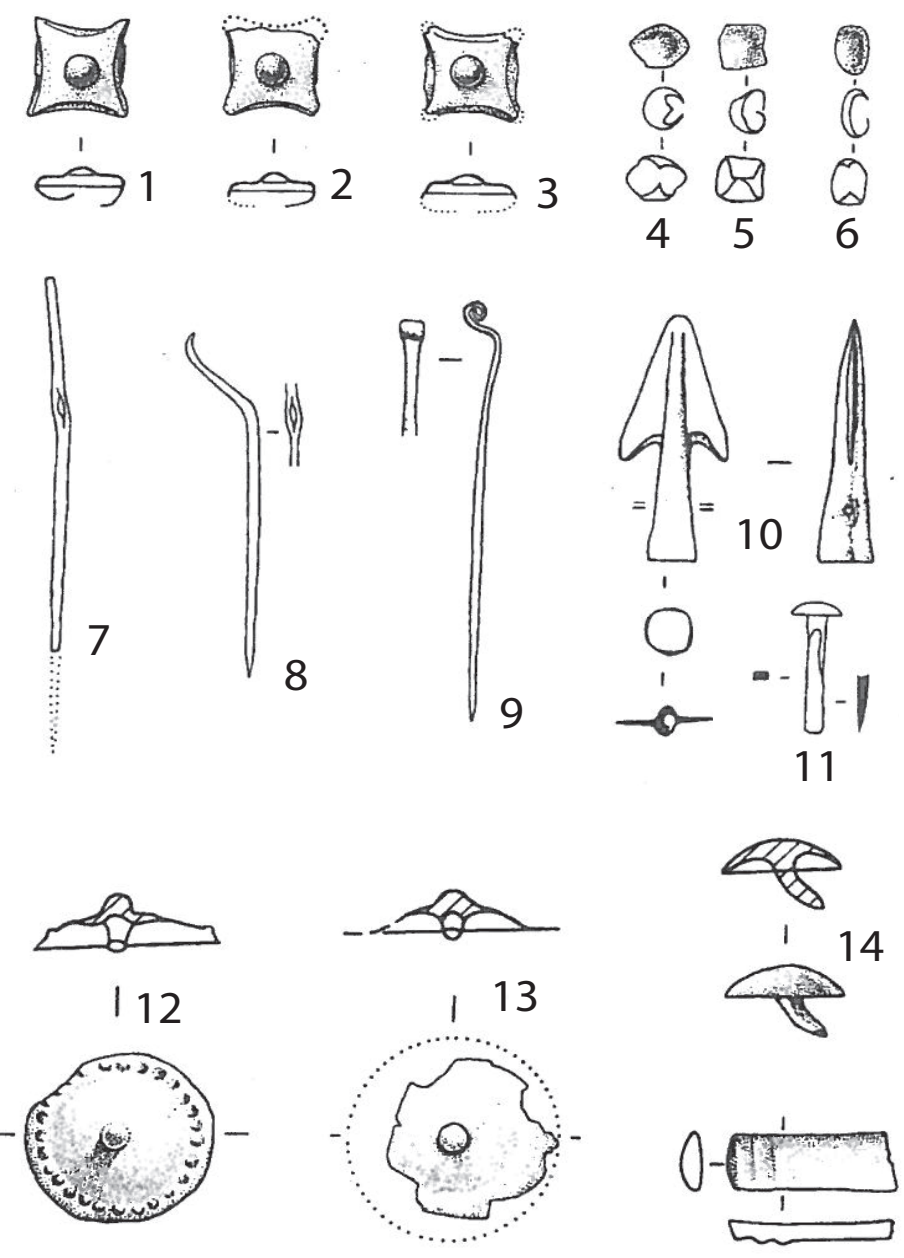

15

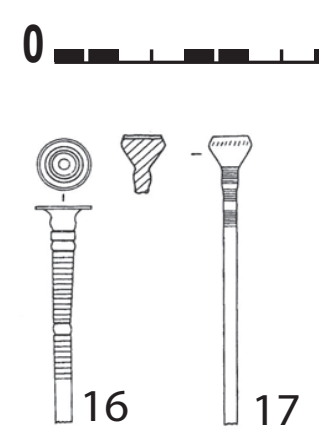

$5 \mathrm{~cm}$

Fig. 2 - Mobilier métallique du domaine de l'Étoile et du col Saint-Anne, Simiane-Collongue, Bouches-du-Rhône (1-17 : d'après Lagrand, 1987; 18-20 : d'après Congès, 1982). 1-11 : couche 4;12-15: couche 5 ; 16-20 : hors stratigraphie.

Fig. 2 - Metal artefacts from Domaine de l'Étoile and Col Saint-Anne, Simiane-Collongue, Bouches-du-Rhône (1-17 : after Lagrand, 1987; 18-20: after Congès, 1982). 1-11: layer 4; 12-15: layer 5; 16-20: out of stratigraphy.

dans le massif de l'Étoile. Il fit l'objet de cinq campagnes de fouilles dirigées par C. Lagrand entre 1968 et 1972 (Vital 1999, p. 99). Ce dernier a observé dans le secteur III une stratigraphie intéressant la Protohistoire. Le Bronze final $3 \mathrm{~b}$ y est plus particulièrement documenté par les couches 4 et 5 (Lagrand, 1987), dans lesquelles n'on observe que quelques intrusions du premier âge du Fer. L'extrémité d'un bracelet du groupe d'Orgon, orné de trois bourrelets transversaux, provient de la couche 5 (fig. 2, n ${ }^{\circ} 15$ ). Il y est associé à des appliques hémisphériques à bouton et bélière caractéristiques du Bronze final (fig. 2, $\mathrm{n}^{\text {os }} 12$ et 13). Deux épingles ont également été découvertes hors stratigraphie. La première (fig. 2, $n^{\circ} 16$ ) comporte une tête discoïde ornée qui la rapproche d'exemplaires des tombes 106 et 107 de la nécropole du Moulin à Mailhac (Aude), datées du Bronze final 3b (Taffanel et Janin 1998, fig. 129). La seconde, à tête biconique et sommet concave (fig. 2, $\mathrm{n}^{\circ} 17$ ), serait en revanche plus ancienne, du Bronze final $3 \mathrm{a}$.

À l'occasion de ramassages de surface et du tamisage de déblais provenant de fouilles clandestines, un second fragment d'une extrémité de bracelet muni d'une cannelure sur le jonc (fig. 2, n 20) a également été découvert (Congès, 1982). 


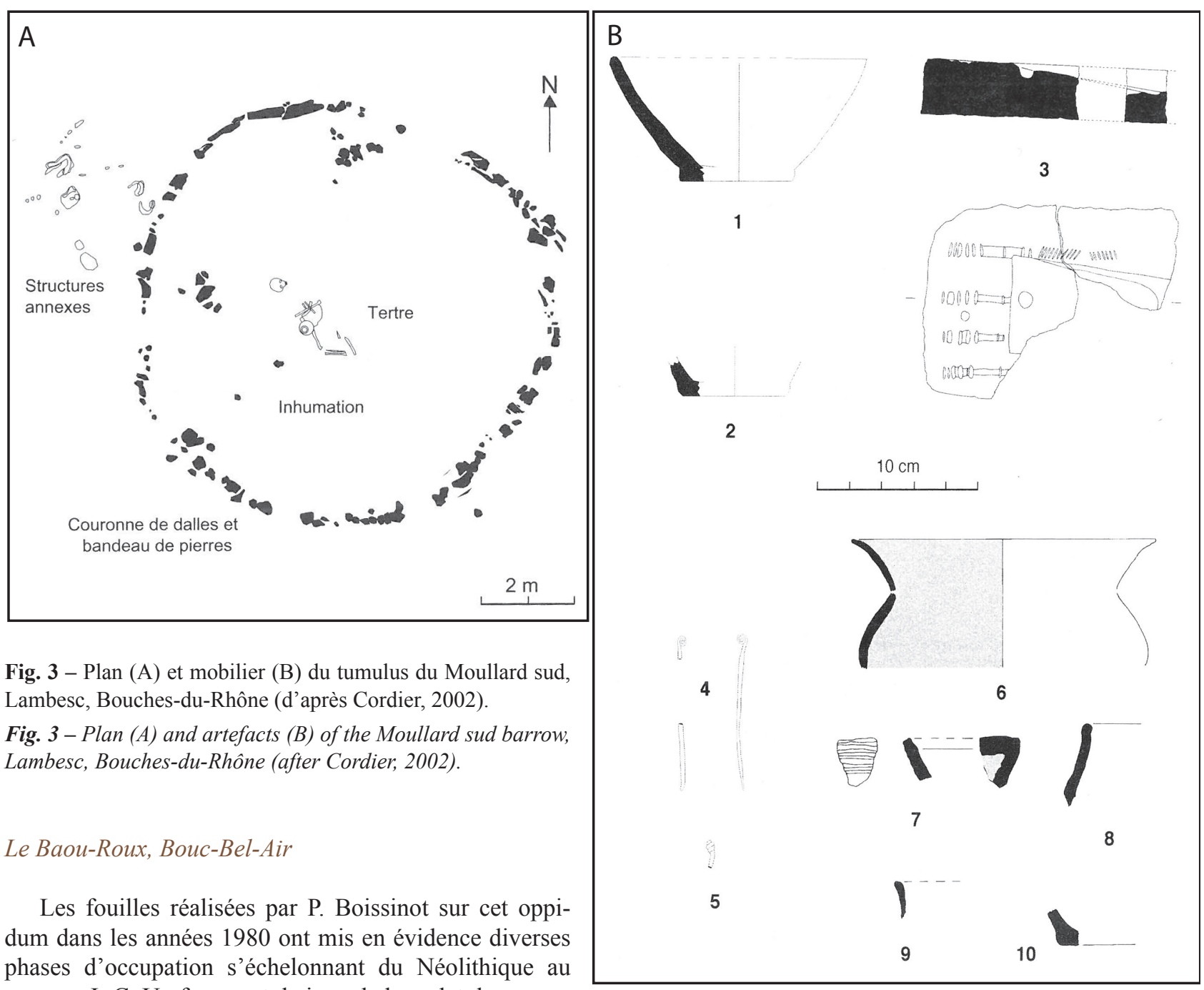

$\mathrm{II}^{\mathrm{e}} \mathrm{S}$. av. J.-C. Un fragment de jonc de bracelet du groupe d'Orgon, orné de deux groupes de cannelures obliques alternées (fig. 1, n ${ }^{\circ}$ 2), appartient à la période 4 de fréquentation du site, datée du Bronze final 3b. Cette phase correspond à des structures d'habitats sur poteaux porteurs, au plan quadrangulaire, et dispersées sur la surface de l'oppidum (Boissinot, 1993).

\section{Le tumulus du Moullard sud, Lambesc}

Le lieu-dit Le Moullard a fait l'objet d'une évaluation, dans le cadre de l'opération de sauvetage archéologique du TGV-Méditerranée, menée par P. Boissinot en 1995, puis d'une fouille sous la direction de L. Cordier en 1996 (Boissinot et al., 1998; Cordier, 2002). Cette dernière a mis en évidence un tumulus de terre, bordé d'une couronne de dalles orthostates (fig. 3A). L'inhumation, localisée au centre du monument, est celle d'un adulte masculin. Ce dernier était accompagné de plusieurs vases, dont certains peints, de deux épingles à tête enroulée et de quatre fragments d'une valve de moule de fondeur (fig. 3B). L'ensemble permet de dater le site du VIII $^{\text {e }}$ s. av. J.-C., soit de la phase de transition entre l'âge du Bronze et l'âge du Fer (Dedet, 2002), ce que tend à confirmer une datation ${ }^{14} \mathrm{C}$ (Lachenal, 2014, p. 215). Le moule, refaçonné pour la réalisation de haches à douille

ronde, servait à l'origine à fabriquer des séries de quatre bracelets à décors en relief constitués de bourrelets transversaux et obliques alternés (fig. $3 \mathrm{~B}, \mathrm{n}^{\circ} 3$ ).

\section{Le dépôt de Notre-Dame de Beauregard, Orgon}

Ce dépôt a été mis au jour dans les années 1970 par M. Calloi, amateur et prospecteur, en contrebas de la chapelle de Notre-Dame de Beauregard, sur l'une des petites terrasses surplombant la Durance. Faiblement enterré (entre $0,20 \mathrm{~m}$ et $0,45 \mathrm{~m}$ de profondeur), il était très probablement contenu dans un récipient en matière périssable. La découverte fut signalée à A. Müller en 1986 (Müller, 2004). L'ensemble comprenait quarante-cinq objets en bronze parmi lesquels on compte une hache à ailerons, une bouterolle en forme de bourse de type atlantique, un élément tubulaire et un fragment de tôle décorée (fig. 4). Les objets de parure sont néanmoins les plus nombreux avec huit phalères décorées de grènetis, utilisés vraisemblablement avec huit boutons coniques à bélière, un tube spiralé, une applique annulaire, quatre bracelets entiers du groupe d'Orgon et dix-neuf fragments de bracelet du même type. Dix exemplaires au moins sont ornés de décors gravés, en grande partie effacés, figurant des 

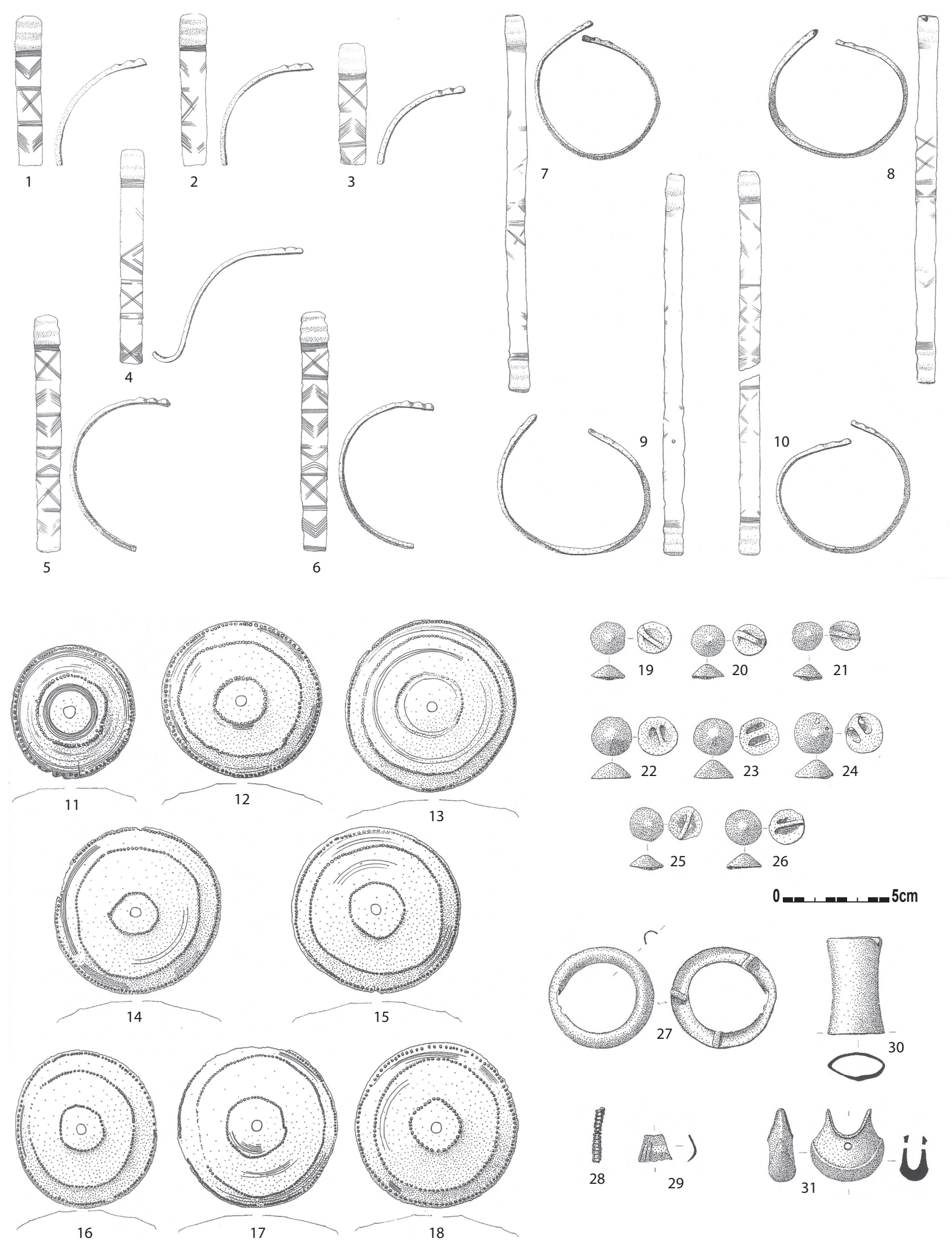

Fig. 4 - Mobilier du dépôt de Notre-Dame de Beauregard, Orgon, Bouches-du-Rhône (d'après Müller, 2004).

Fig. 4 - Objects from the hoard of Notre-Dame de Beauregard, Orgon, Bouches-du-Rhône (after Müller, 2004). 

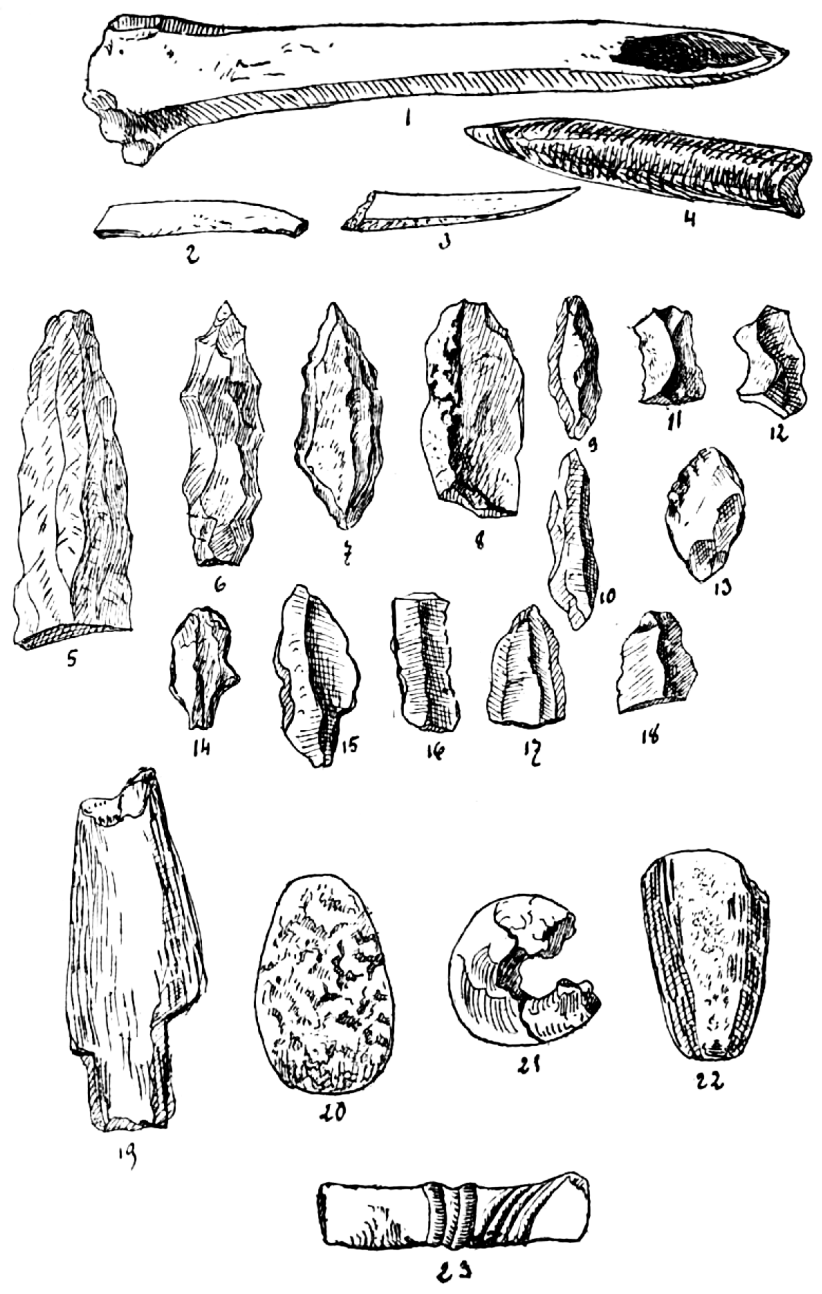

croix de saint André, des chevrons couchés et des lignes parallèles. Leurs extrémités sont soulignées par les trois bourrelets transversaux caractéristiques de cette forme de parure annulaire (fig. 4, $\mathrm{n}^{\text {os }} 1-10$ ).

Les objets de ce dépôt, en particulier la bouterolle en bourse (fig. $4, \mathrm{n}^{\circ} 31$ ) correspondant au type A1 de P-Y. Milcent (2009, fig. 6), permettent de dater l'ensemble du Bronze final $3 \mathrm{~b}$.

\section{Carrière de Bruny, Berre-l'Étang}

Ce site menacé par une carrière à fait l'objet de fouilles au début du XX $\mathrm{X}^{\mathrm{e}}$ s. par J. Cotte (1924). Ce dernier y découvrit les restes d'une occupation semblant se rapporter en grande partie au Néolithique, des sépultures et un unique objet métallique. Ce dernier, interprété à l'origine comme un outil, semble correspondre à un fragment de jonc de bracelet du groupe d'Orgon orné de reliefs transversaux et obliques alternés (fig. $5, \mathrm{n}^{\circ} 23$ ).

\section{«Ossuaire no 1 » de Romanin, Saint-Rémy- de-Provence}

Cette grotte fouillée par le commandant Thoret au début du $\mathrm{XX}^{\mathrm{e}} \mathrm{s}$. (Rolland, 1938) a servi de sépulture col-
Fig. 5 - Mobilier de la Carrière de Bruny, Berre-l'Étang, Bouches-du-Rhône (d'après Cotte, 1924).

Fig. 5 - Artefacts from the Carrière of Bruny, Berre-l'Étang, Bouches-du-Rhône (after Cotte, 1924).

lective à une période indéterminée. Le mobilier récolté lors des fouilles suggère des occupations du Néolithique final, de l'âge du Bronze et de l'âge du Fer. Le seul objet en bronze découvert est un bracelet ouvert aux extrémités ornées de bourrelets transversaux assimilable au groupe d'Orgon (fig. 6, $\mathrm{n}^{\circ} 11$ ). Si le décor de cette pièce n'est pas visible sur la photographie accompagnant la publication, il y est décrit comme formé de « traits parallèles gravés » (Rolland, 1938, p. 472).

\section{Vaucluse}

\section{La nécropole des Lauzières, Lourmarin}

Découvert en 1965, le site des Lauzières à Lourmarin a fait l'objet de sept campagnes de fouilles annuelles entre 1976 et 1982 sous la direction de J. Courtin. Les objectifs de l'opération étaient l'analyse d'un habitat du Néolithique final comportant un mur d'enceinte barrant l'éperon portant le site. À proximité de cet aménagement, un ensemble funéraire de l'âge du Bronze a été mis au jour. Il comporte onze ensembles sépulcraux, vraisemblablement des inhumations secondaires sous tumulus (D'Anna

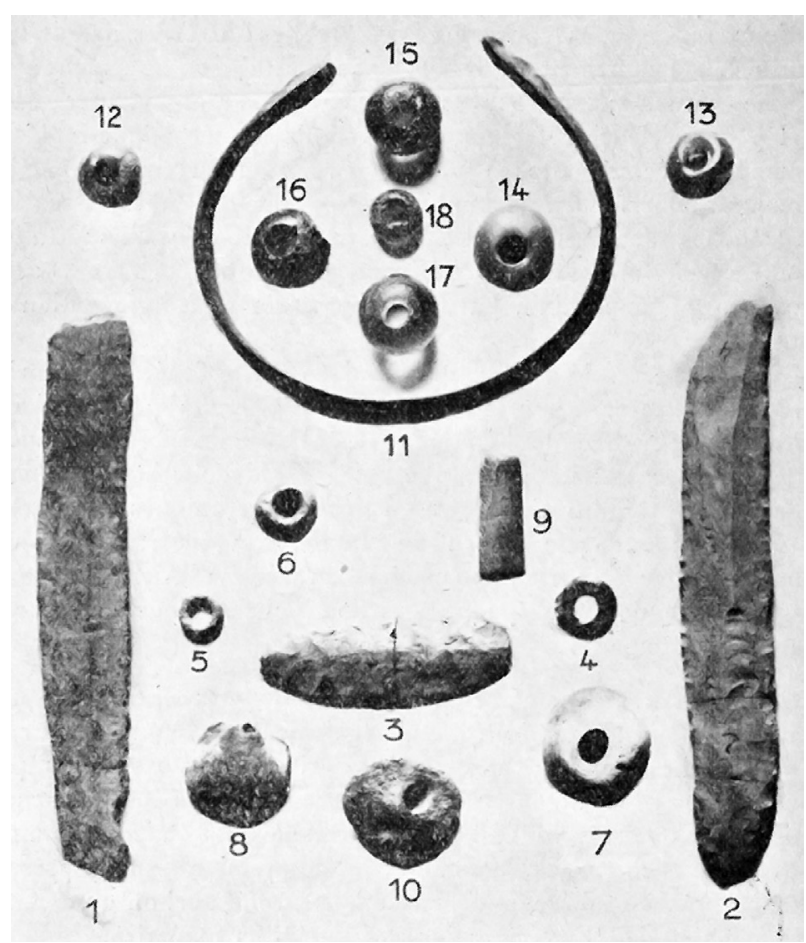

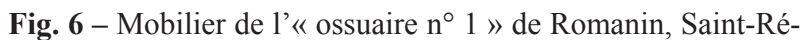
my de Provence, Bouches-du-Rhône (d'après Rolland, 1938).

Fig. 6 - Artefacts from the " ossuaire $n^{\circ} 1$ », Romanin, SaintRémy-de-Provence, Bouches-du-Rhône (after Rolland, 1938). 

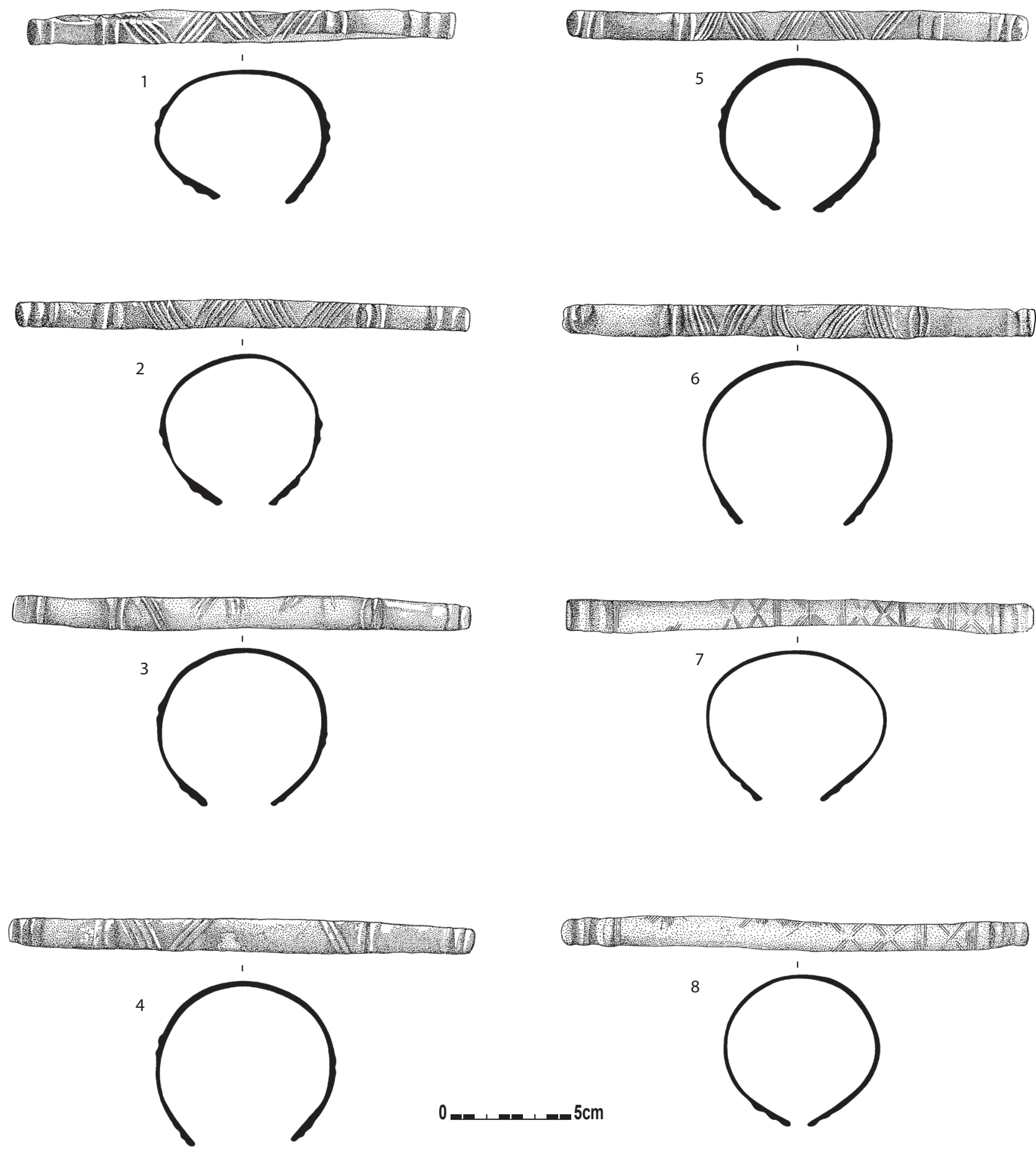

Fig. 7 - Bracelets de la nécropole des Lauzières, Lourmarin, Vaucluse (d'après D'Anna et al., 2002).

Fig. 7 - Bracelets from the Lauzières necropolis, Lourmarin, Vaucluse (after D'Anna et al., 2002).

et al., 2002). Cette occupation a livré douze parures annulaires attribuables au groupe d'Orgon, dont dix aux moins proviennent de l'ensemble 3. Parmi ces dernières, quatre bracelets et un fragment portent un décor gravé alternant des motifs cruciformes et des lignes transversales (fig. 7, $\mathrm{n}^{\text {os }} 7$ et 8 ). Les cinq autres possèdent une ornementation obtenue lors du moulage, composée de bossettes horizontales et de groupes de nervures obliques alternées ${ }^{(2)}$ (fig. 7, $n^{\text {os }} 2-6$ ). Un autre bracelet légèrement plus court est en revanche de provenance stratigraphique inconnue (fig. 7, no 1). Enfin, un dernier exemplaire provient de l'ensemble 10, il est décrit comme terminé par des renflements et décoré d'incisions très fines et irrégulières « disposées en quatre séries de chevrons opposés par la base déterminant des losanges et triangles réservés » (documentation A. D'Anna).

L'ensemble 3 a également livré une épingle assez atypique proche des exemplaires à tête vasiforme, un anneau, une bague spiralée et huit bagues en tôle ornées de cannelures. Des objets simi- 
laires se retrouvent dans le dépôt de Moriez dans les Alpes-de-Haute-Provence (Barge, 2004) ainsi que dans la tombe de Maison de Torrenté à Sion en Suisse (David-Elbiali et Dunning, 2005, pl. $15 \mathrm{n}^{\circ}$ 5). L'association de ce type de bracelet à l'épingle permet donc de dater la sépulture de l'ensemble 3 du Bronze final 3b. Cette dernière n'a livré que des fragments de crâne et de mandibule appartenant à un individu adulte âgé (D’Anna et al., 2002, p. 217).

\section{La grotte du Passage, Buoux}

La grotte du Passage compte parmi les nombreux gisements karstiques de la vallée de l'Aiguebrun occupés durant la Protohistoire. Découvert en 1965 par J. Courtin, elle a fait l'objet de travaux de la part de ce dernier en parallèle à la fouille d'autres cavités du Chaos de Buoux (Vital, 2002a, p. 84). Un ensemble de six bracelets identiques ont été découverts dans une trémie emplie de blocs de l'une des salles de la grotte (fig. 1, $\mathrm{n}^{\circ} 4$ ). Ces derniers portent un décor gravé très corrodé. Sur l'exemplaire le mieux conservé il s'agit d' « une suite de triangles incisés alternes encadrés aux extrémités par des séries d'incisions transversales ellesmêmes séparées par des motifs cruciformes » (Vital, 2002b, p. 213). En outre, la cavité a livré des récipients attribués au Bronze moyen ainsi que des ossements humains. La nature funéraire des bracelets n'est cependant pas assurée et l'ensemble pourrait correspondre à un dépôt, tel qu'il est connu à proximité dans la grotte des Épingles à Cheval-Blanc (Vital, 2002a, p. 88-89 et 2002c, fig. 28 nos $^{\text {os }}$ et 3).

\section{"Orange »}

De la collection Vallentin du Cheylard à Montélimar (Drôme) provient un bracelet, orné de lignes transversales et obliques réalisées à la coulée, du groupe d'Orgon (fig. 1, $\left.\mathrm{n}^{\mathrm{o}} 3\right)^{(3)}$. La seule indication de provenance est une étiquette associée à l'objet mentionnant Orange, comme de nombreuses pièces de cette collection privée. Dès lors, il paraît vraisemblable qu'il s'agisse du lieu d'achat et non de découverte de cet objet.

\section{Ardèche}

\section{Nécropole tumulaire de Beauregard, Saint-Remèze}

Cette nécropole d'une vingtaine de tertres a été partiellement fouillée dans les années 1950-1960. Elle livre plusieurs ensembles tumulaires pour lesquels les associations d'objets ne sont pas assurées. Plusieurs contextes montrent ainsi l'association de mobiliers du premier âge du Fer, parmi lesquels de la céramique grise monochrome des $\mathrm{VI}^{\mathrm{e}}-\mathrm{V}^{\mathrm{e}} \mathrm{S}$. av. J.-C., avec des objets du Bronze final 3b. Il s'agit notamment d'une épingle à tête vasiforme du tumulus 7 et de deux vases en céramique non tournée des tertres 8 et 13 (Lagrand, 1987, p. 51-52; Dedet et Durand, 2016). Cette situation peut découler du mélange de mobilier provenant de tombes différentes ou de l'utilisation successive de plusieurs monuments. Au total, huit bracelets à extré- mités côtelées proviennent de ce site. L'un d'entre eux, rattaché au tumulus 1 , possède un décor gravé associant, aux extrémités, deux motifs cruciformes séparés par des lignes transversales, tandis que la partie centrale du jonc est ornée de trois ovales étirés imbriqués (fig. 8, $\mathrm{n}^{\circ} 1$ ). Deux autres exemplaires, qui provennent du tumulus 4 et dont les décors ont été réalisés selon la même technique, supportent des motifs de chevrons imbriqués et affrontés, alternés avec des panneaux de lignes transversales (fig. 8, $\mathrm{n}^{\circ} 2$ et 3 ). Le tertre 7 livre également quatre bracelets identiques dont le jonc est orné de trois motifs ovalaires imbriqués et les extrémités soulignées par des lignes gravées (fig. 8, $\mathrm{n}^{\circ} 4$ ). Une dernière parure annulaire, semblable aux précédentes, n'a, pour sa part, pas pu être associée à une tombe en particulier (fig. $8, \mathrm{n}^{\circ} 5$ ).

\section{Dépôt de la grotte du Déroc, Vallon-Pont-d'Arc}

Ce dépôt d'objets du Bronze final 3b avait été placé dans un vase recouvert d'une pierre plate. Il fut découvert à l'intérieur de la cavité du Déroc à Vallon-Pont-d'Arc, alors exploitée comme carrière de phosphate (Ollier de Marichard, 1884a et 1884b; Roudil, 1990; Gascó et Borja, 2016). Si la composition exacte de l'ensemble est difficile à établir, il pouvait comprendre près de 300 pièces, principalement des éléments de parure. Le musée de Nîmes conserve actuellement 250 objets parmi lesquels on compte des pendeloques, anneaux, boutons à bélières, sphéroïdes et bracelets en bronze, ainsi qu'une parure annulaire en roche noire, une pièce en cuir et un cordon en boyau. La présence de parures métalliques avec des éléments organiques ou minéraux (des perles en ambre et une dent d'ours faisaient également partie du lot lors de sa découverte), rapproche cet ensemble des dépôts du type de Blanot (Verger, 1998), dont la composition évoque de riches costumes individuels féminins, connus en France dès le Bronze final $2 b$ et jusqu'au début du premier âge du Fer à la Motte à Agde (Verger et al., 2007).

Les bracelets métalliques de ce dépôt, au nombre de treize à l'origine, comptent actuellement sept exemplaires conservés. Quatre d'entre eux se rapportent au groupe d'Orgon avec deux bourrelets transversaux aux extrémités. Ces objets portent des décors gravés de croix de saint André associés à un motif rhomboïdal ou ovalaire constitué de lignes incisées et hachurées. D'autres éléments en relief, côtes ou bossettes, sont également disposés vers l'extérieur du jonc (fig. 9).

\section{Bérias-et-Casteljau}

Le musée Calvet d'Avignon conserve deux bracelets identiques ( $\mathrm{n}^{\circ}$ d'inv. 252 B) provenant de Casteljau (Campolo et Garcia, 2004, p. 67, $\mathrm{n}^{\text {os }} 106$ et 107). Bien que les circonstances exactes de cette découverte ne soient pas connues, le fait que l'un d'entre eux soit plié laisse penser qu'ils provenaient à l'origine d'une sépulture ou d'un dépôt. Leurs extrémités sont soulignées de bourrelets transversaux et leur jonc est orné de deux ovales étirés gravés (fig. $1, \mathrm{n}^{\circ} 5$ ). 


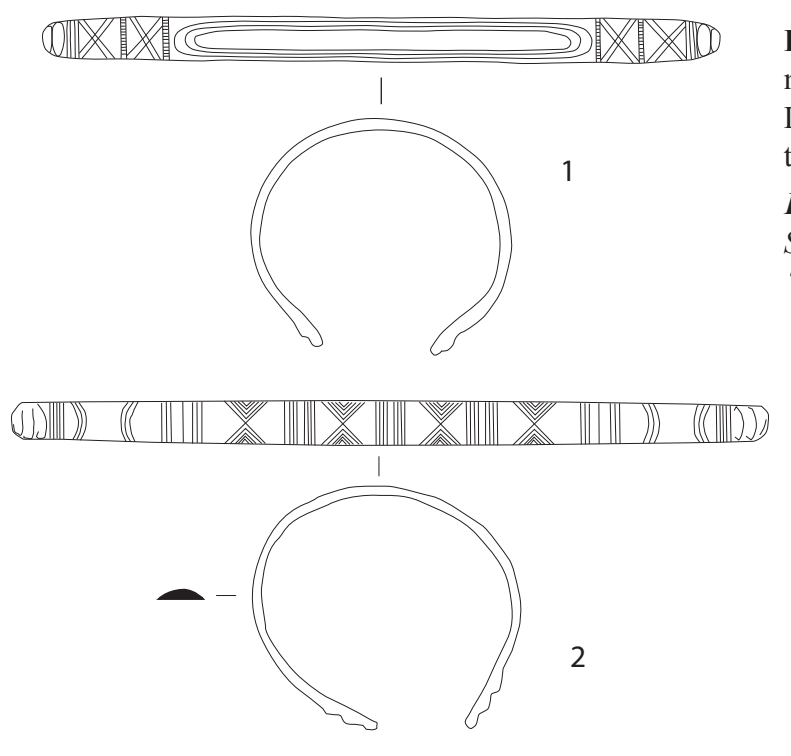

Fig. 8 - Bracelets du groupe d'Orgon provenant de la nécropole tumulaire de Beauregard à Saint-Remèze, Ardèche (d'après Dedet et Durand, 2016). 1, tumulus 1 dit de l'Arbre Rond. 2-3, tumulus 4. 5, tumulus 7.6, tumulus non précisé.

Fig. 8 -Bracelets of Orgon group from the Beauregard necropolis, Saint-Remèze, Ardèche (after Dedet and Durand, 2017). 1: barrow $n^{\circ} 1$ 'l'Arbre Rond'; 2-3: barrow no 4; 5: barrow no 7; 6: unknown barrow.
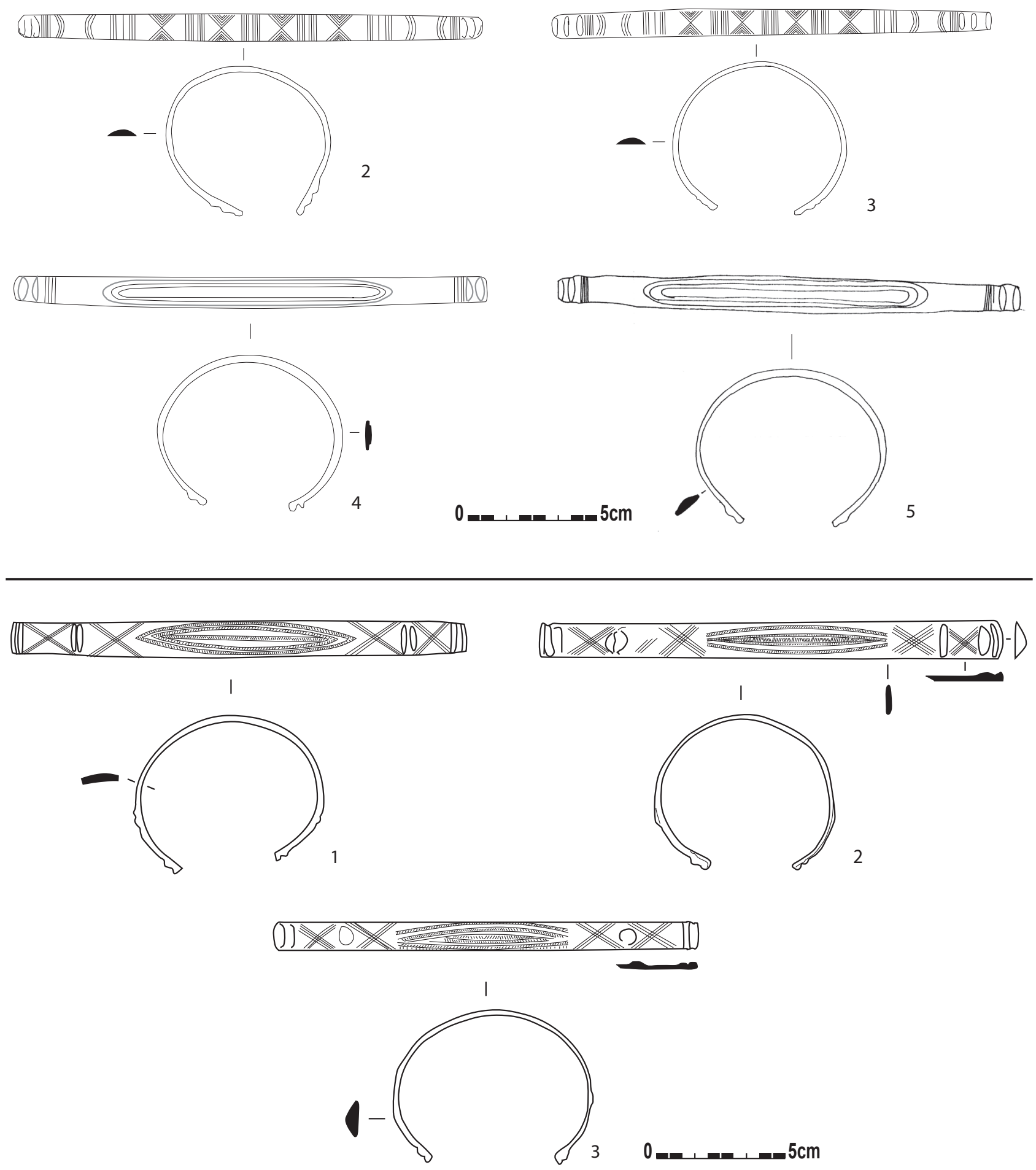

Fig. 9 - Bracelets du groupe d'Orgon du dépôt de la grotte du Déroc à Vallon-Pont-d'Arc, Ardèche (d'après Gascó et Borja, 2016, fig. 3).

Fig. 9 - Bracelets of Orgon group from the hoard of the Déroc cave, Vallon-Pont-d'Arc, Ardèche (after Gascó and Borja, 2016, fig. 3). 


\section{ÉTUDE TYPOLOGIQUE}

\section{Types décoratifs}

Les bracelets appartenant au groupe d'Orgon présentent des décors couvrant la totalité du jonc. Une certaine variabilité de ces ornementations peut être décelée en fonction de la technique utilisée et des motifs représentés. Les décors plastiques, en relief, correspondent notamment à des côtes ou des bossettes assez proches des bourrelets transversaux ornant les extrémités de ces parures. Ils sont généralement localisés au premier quart de la demi-longueur de l'objet. Ces éléments peuvent être associés à des décors gravés ou également en relief. Dans ce cas il s'agit alors uniquement de groupes de côtes obliques, dont le nombre varie entre quatre et dix et dont le sens d'exécution est alterné. Les décors graphiques gravés présentent pour leur part une variété plus importante de motifs, des lignes transversales, mais aussi des croix, des triangles hachurés ou des chevrons imbriqués et des ovales étirés.

La combinaison de ces différents décors permet de définir trois types de bracelets du groupe d'Orgon (fig. 10).
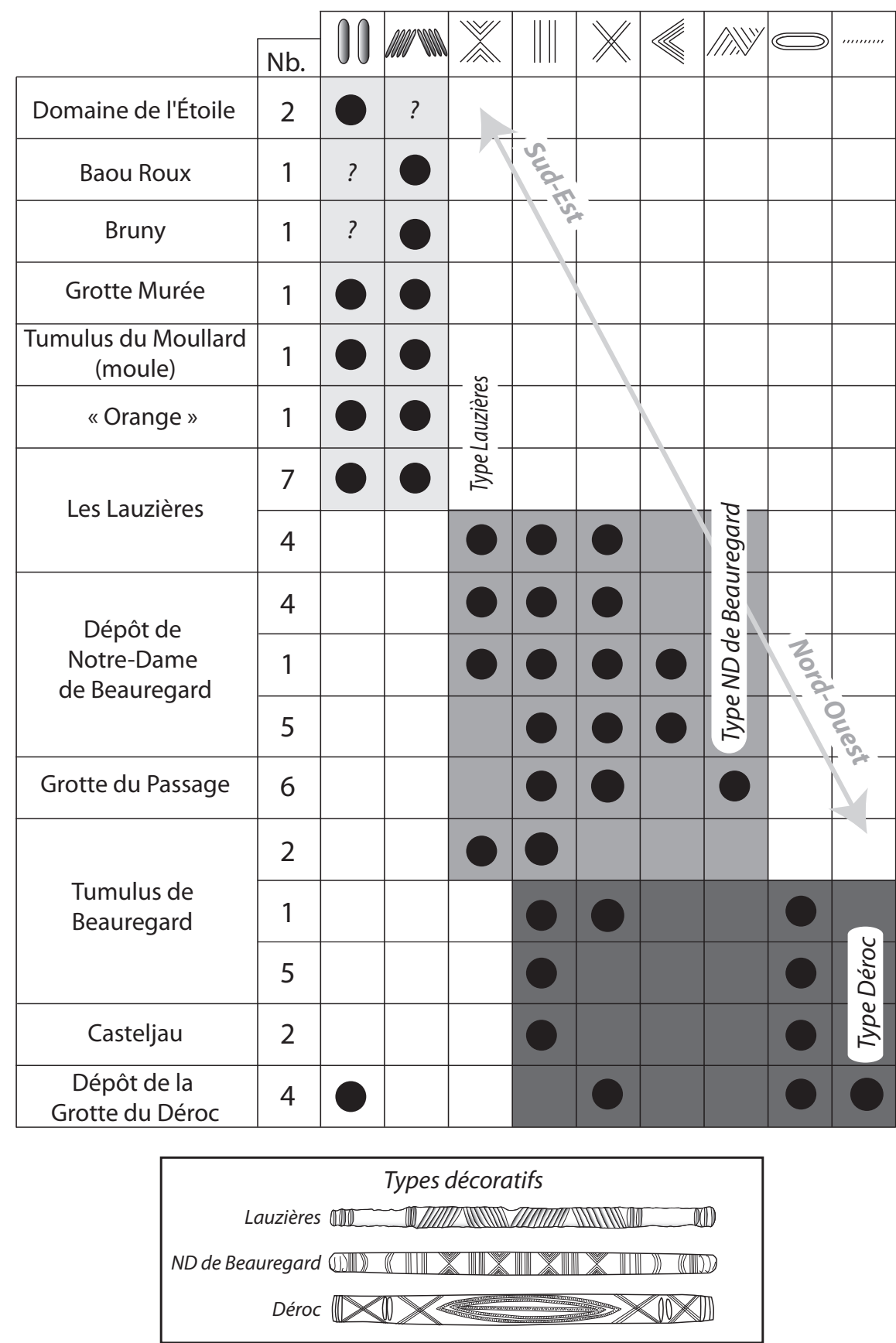

Fig. 10 - Classification des types décoratifss des bracelets du groupe d'Orgon en fonction des motifs représentés.

Fig. 10 - Classification of the decorative types of the bracelets of Orgon group according to the patterns depicted. 
Le premier, présent en plus grand nombre aux Lauzières (fig. 7, $\mathrm{n}^{\text {os }} 1-6$ ), correspond aux exemplaires supportant uniquement un décor en relief. Il est également présent au Baou Roux (fig. 1, nº 2), à Bruny (fig. 5, n 23), à la grotte Murée (fig. 1, $\mathrm{n}^{\circ} 1$ ), sur l'exemplaire d'Orange (fig. $1, \mathrm{n}^{\circ} 3$ ), et il est probable que les fragments du domaine de l'Étoile, sur lesquels aucun décor gravé n'est conservé, s'y rapportent également (fig. 2, $\mathrm{n}^{\text {os }} 15$ et 20). Ce décor est obtenu lors de la coulée, comme en témoigne le moule du tumulus du Moullard qui a probablement servi à la production de bracelets similaires (fig. $3 b, n^{\circ} 3$ ). Nous appellerons ce type « type des Lauzières ».

Le second type se caractérise par des décors gravés figurant des lignes, mais également des croix et des chevrons, imbriqués ou hachurés. Il est particulièrement bien représenté dans le dépôt d'Orgon avec dix exemplaires (fig. $4, \mathrm{n}^{\text {os }} 1-10$ ), mais on en compte également plusieurs aux Lauzières (fig. $7, \mathrm{n}^{\text {os }} 7$ et 8 ), dans la grotte du Passage (fig. 1, $\mathrm{n}^{\circ} 4$ ) et la nécropole de Beauregard (fig. 8, $\mathrm{n}^{\mathrm{os}} 2$ et 3). Nous appellerons ce type « type de Notre-Dame de Beauregard » pour éviter les confusions avec la dénomination du groupe.

Enfin, un troisième type, également déterminé par la présence d'ornementations gravées, associe les décors cruciformes avec des motifs ovoïdes couvrant le centre du jonc du bracelet. Outre le dépôt du Déroc, qui en livre quatre exemplaires (fig. 9), il se retrouve à Casteljau (fig. 1, $\mathrm{n}^{\circ}$ 5) et dans des tumulus de Beauregard (fig. 8, $\mathrm{n}^{\mathrm{os}} 1,4$ et 5 ). Nous appellerons ce type « type du Déroc ».

\section{Morphologie}

Ces types décoratifs trouvent certaines correspondances avec la morphologie des pièces, si l'on prend en compte leur longueur déroulée et leur largeur maximale, mesurée pour les pièces complètes uniquement (fig. 11). Les objets classés dans le type des Lauzières constituent ainsi un groupe relativement homogène comptant parmi les plus grands exemplaires. Les parures du type du Déroc sont en revanche plus ramassées que la majorité des autres. Enfin, les bracelets se classant dans le type de Notre-Dame de Beauregard présentent une plage de longueurs plus étendue et des exemplaires généralement plus étroits que les autres.

D'un point de vue général, on peut déceler trois classes de longueurs dont les limites se situent vers 160 et $185 \mathrm{~mm}$. Ces dernières pourraient correspondre aux diamètres croissants de bracelets portés en série.

\section{CONTEXTES DE DÉCOUVERTES}

$\mathrm{B}$ ien que pour quelques découvertes anciennes, le contexte de découverte des objets ne soit pas clairement identifié (Bruny, Romanin, Casteljau et Orange), les parures annulaires examinées proviennent à la fois d'habitats, de sépultures et de dépôts non funéraires (tabl. 1 et fig. 12). La première modalité concerne uniquement des sites localisés au Sud-Est de l'aire de découverte de ces bracelets : la grotte Murée, le Baou-Roux et le Domaine de l'Étoile. Les exemplaires qui en proviennent sont des fragments, perdus dans les lieux d'habitations ou préparés pour la refonte. Les découvertes funéraires proviennent notamment des nécropoles tumulaires de Saint-Remèze et de Lourmarin. Dans l'ensemble 10 des Lauzières, ainsi que vraisemblablement dans le tumulus 1 de Beauregard, ils ont été retrouvés isolés. En revanche, l'ensemble 3 des Lauzières en livre dix exemplaires associés à un seul individu. De même, les tertres 4 et 7 de la nécropole de SaintRemèze associent respectivement des ensembles de deux et quatre bracelets identiques, qui devaient appartenir à un même défunt.

Les dépôts du Déroc et de Notre-Dame de Beauregard rassemblent également plusieurs exemplaires du même type. Au Déroc, ils font partie d'un ensemble d'au moins quatorze bracelets en métal et roche noire qui devaient participer du costume d'un seul individu. La surreprésentation des parures dans ce dépôt lui confère par ailleurs une connotation féminine. Le dépôt de Notre-Dame de Beauregard présente en revanche une composition plus hétéroclite, qui associe également des éléments d'armements tels qu'une bouterolle et une possible pièce de poignée d'épée. Les bracelets y ont majoritairement été découverts sous forme de fragments. Il est donc difficile de définir s'ils appartenaient avec les autres éléments à une unique panoplie masculine, si le dépôt associe deux lots d'objets de sphères différentes, à l'image des ensembles du type du Petit-Villatte à Neuvy-surBarangeon (Milcent, 1998), ou si ces fragments de bracelets constituent une réserve de métal, ici associée à des éléments d'épées comme dans les dépôts du type de Brülh-Briod (Verger, 1992). Un autre lot de six bracelets identiques provient de la grotte du Passage. Si une fonc-

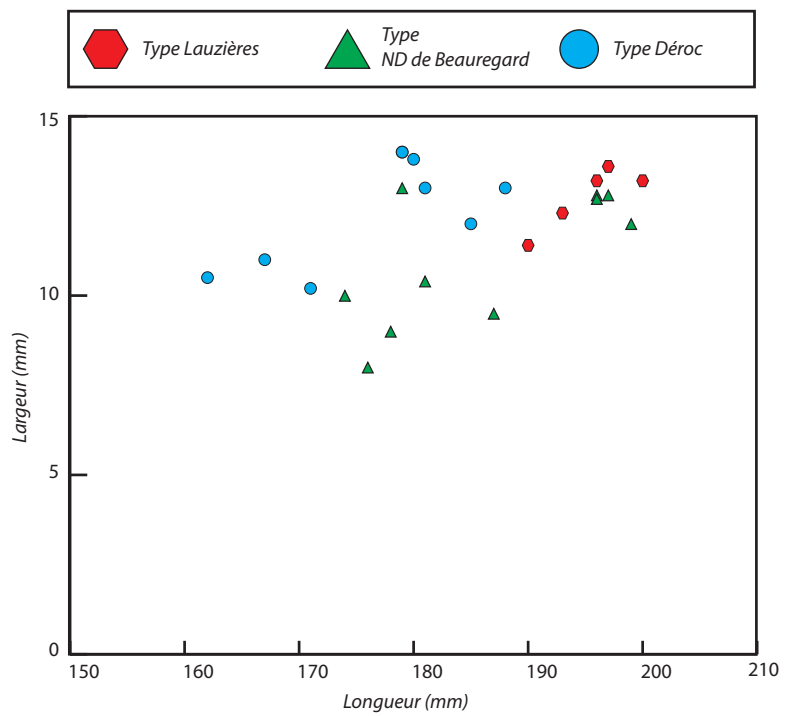

Fig. 11 - Diagramme de corrélation entre la longueur et la largeur du jonc des bracelets entiers.

Fig. 11 - Length/width scatter diagram of the whole bracelets. 


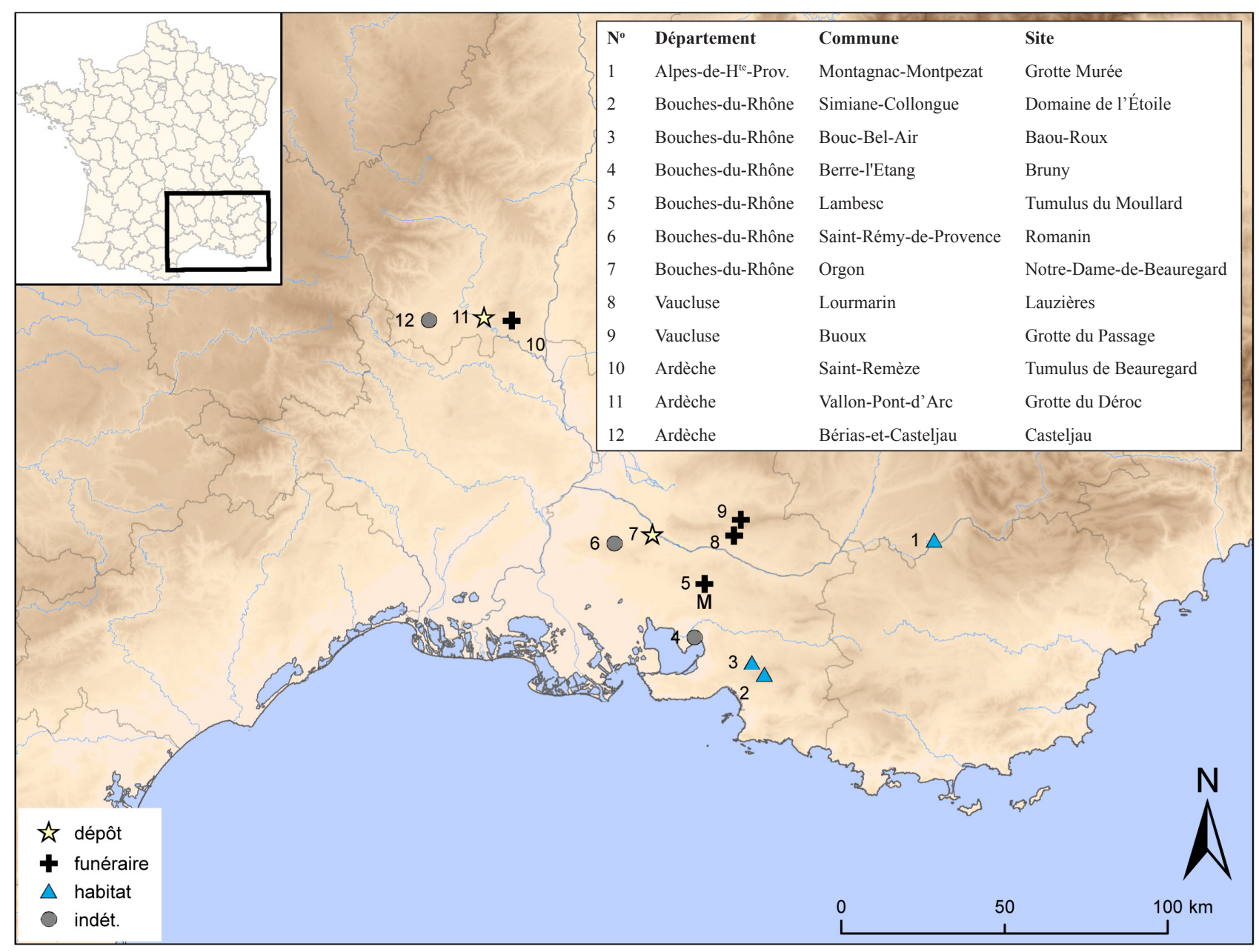

Fig. 12 - Répartition des bracelets du groupe d'Orgon en fonction du contexte de découverte.

Fig. 12 - Distribution of the bracelets of Orgon group according to the finding context.

tion sépulcrale de cet ensemble semble la plus probable, on ne peut exclure qu'il s'agisse d'un dépôt non funéraire en grotte du type de celui du Déroc. Cette notion de série est également suggérée par le moule de Lambesc permettant de couler quatre bracelets simultanément.

Il ressort de cet inventaire que les bracelets du groupe d'Orgon sont probablement des objets du quotidien, retrouvés à l'état de fragments sur les sites d'habitat, mais qu'ils peuvent également participer de parures beaucoup plus ostentatoires, à vocation peut être cérémonielle comme l'évoque costume richement orné de la grotte du Déroc. Les découvertes sépulcrales indiquent qu'ils ont pu être portés soit isolément, soit en série. Les lots provenant de l'ensemble 10 des Lauzières et des deux dépôts illustrent bien ce cas de figure, avec des longueurs croissantes permettant d'épouser la forme de l'avant-bras (fig. 13). Les deux séries de types décoratifs présentes aux Lauzières pouvaient d'ailleurs orner chacune un bras de l'individu. Le mode de dépôt secondaire des restes humains dans cette sépulture ne permet pas de connaître le sexe de l'individu ayant possédé ces parures. Les deux modalités de port, isolément et en série, pourraient peut-être correspondre respectivement aux genres masculin et féminin. À moins que cette différenciation ne soit un marqueur social. Par exemple, dans l'Aurès (Algérie), les femmes âgées portent un plus grand nombre de bracelets que les plus jeunes, tandis que les fillettes ne parent leurs poignets que d'un ou deux anneaux (Benfoughal, 1998, p. 165).

\section{CHRONOLOGIE ET ORIGINE DU GROUPE}

$\mathrm{L}$ es contextes de découvertes les mieux datés convergent vers une attribution de ces bracelets au Bronze final $3 b$, correspondant principalement au $\mathrm{IX}^{\mathrm{e}} \mathrm{S}$. av. J.-C. Une fois n'est pas coutume, plusieurs sites d'habitats concourent à la datation de ces objets métallique, qui y sont associés à des ensembles céramiques bien caractérisés, comme au Baou-Roux et au Domaine de l'Étoile. Cette datation est confirmée par la typologie des objets des dépôts du Déroc et de Notre-Dame de Beauregard, ainsi que par les autres objets métalliques des Lauzières. Par ailleurs, des sites au contexte moins assuré témoignent d'une occupation de cette période, tels que la grotte Murée et probablement la nécropole 


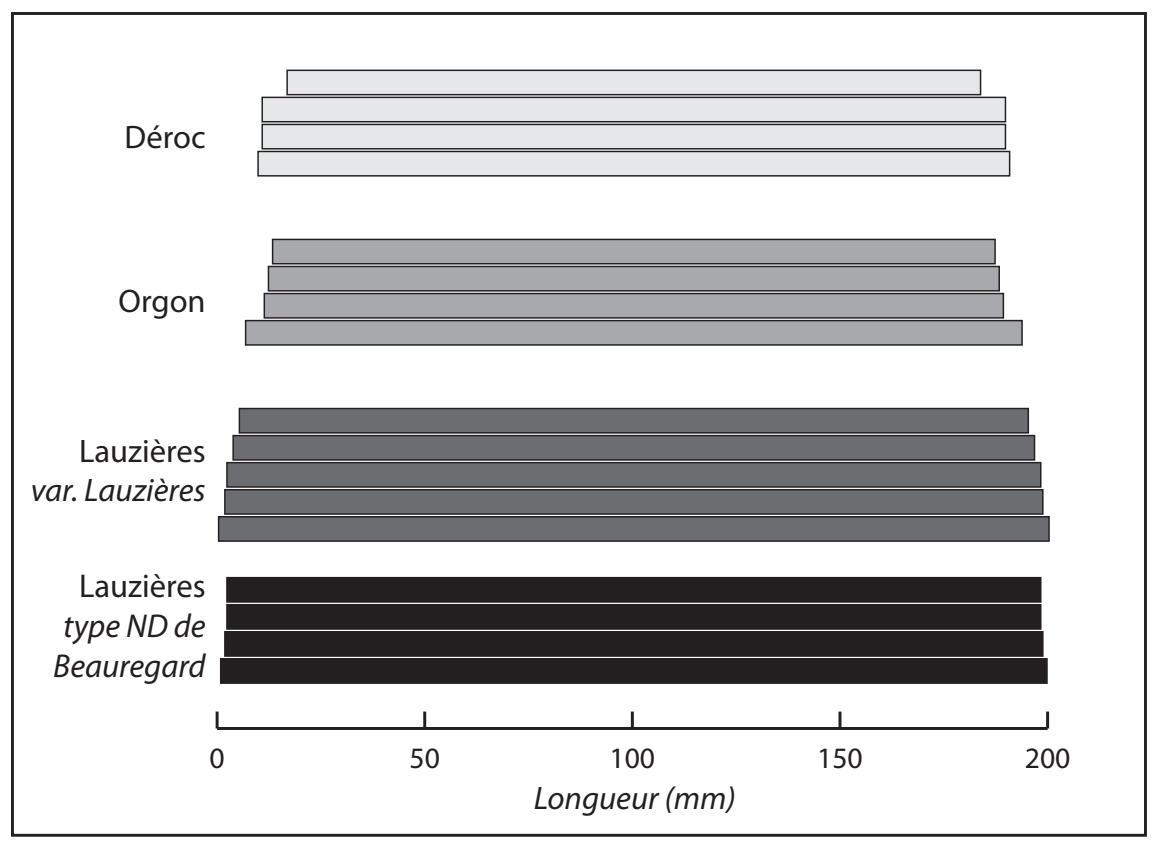

Fig. 13 - Histogramme des longueurs des bracelets complets pour les sites ayant livré plusieurs individus.

Fig. 13 - Histogram of the lengths of the whole bracelets from the contexts with several objects.

de Beauregard. La datation la plus récente est fournie par le moule du tumulus du Moullard sud, attribué à la phase de transition Bronze-Fer de la chronologie méridionale, au sens de T. Janin (1992), correspondant au Hallstatt C ancien (Milcent 2009, fig. 10). Mais il s'agit d'un objet refaçonné, ne donnant dès lors qu'un terminus ante quem.

L'origine de cette forme de parure peut être recherchée dans les bracelets du type de Pourrières, présentant une section un peu plus massive, le plus souvent triangulaire, mais la rejoignant dans leurs décors gravés et leurs bossettes aux extrémités et parfois au premier quart de leur demi-longueur. Ce type est bien représenté dans le Sud-Est de la France au Bronze final $2 \mathrm{~b}$ et $3 \mathrm{a}$, dans le dépôt éponyme de la Farigourière dans le Var (Courtois, 1957) mais aussi dans ceux de L'Épine et de Réallon dans les Hautes-Alpes (Haussmann, 1996-1997). Un fragment brut de coulée associé à une tuyère coudée sur le site du Bastidon à Sillans-la-Cascade dans le Var atteste une production locale de ce type (Lachenal, 2011, p. 253). Bien représenté dans les Alpes françaises, on en connait quelques exemplaires sur la façade atlantique et en Languedoc, comme à Carlipa dans l'Aude (Éluère et Gomez, 1990, p. 61 ; Ricalens et Soutou, 1965). L'ornementation des exemplaires à décor en relief évoque également celle des bracelets du type de Publy, daté du début du Bronze final (Éluère et Gomez 1990, p. 45). Cette morphologie est surtout connue dans le quart nord-est de la France, mais une série fait également partie du dépôt de Cabanelle à Castelnau-Valence dans le Gard (Dedet et Bordreuil, 1982).

Parmi les contextes contemporains, quelques exemplaires à extrémités côtelées, qui pourraient avoir servi de modèles aux bracelets du groupe d'Orgon, sont présents dans la métallurgie atlantique de la fin du Bronze final. Il s'agit de parures annulaires ouvertes du type de Vénat (Éluère et Gomez, 1990, p. 55) qui, bien que de section plus lenticulaire ou franchement circulaire, peuvent présenter des décors gravés tout à fait similaires, par exemple à Choussy (Loir-et-Cher) ou au Petit-Villatte à Neuvysur-Barangeon dans le Cher (Cordier, 2009, fig. $201 \mathrm{n}^{\text {os }} 3$ et 10 , fig. $241 \mathrm{n}^{\text {os }} 1$ et 8 ). Un fragment de section aplanie, plus proche des exemplaires méridionaux, est également présent dans le dépôt de Meschers en CharenteMaritime; il est néanmoins orné d'un décor poinçonné (Gachina et al., 2008, fig. 3 no 38). D'autres bracelets à extrémités moulurées proviennent du tertre de Saubert à Hures-la-Parade (Lozère). Ils sont associés à des parures annulaires du premier âge du Fer (Dedet, 2001, p. 161, fig. 174 b et $174 \mathrm{c})$.

Le groupe d'Orgon pourrait donc procéder d'une évolution locale, éventuellement teintée d'inspirations atlantiques. Plusieurs indices permettent cependant de caractériser une production et une diffusion principalement régionale de ces parures.

\section{PRODUCTION ET DIFFUSION}

\section{Répartition géographique}

La cartographie des découvertes de bracelets du groupe d'Orgon montre une extension très limitée de cette forme de parure, qu'on ne trouve exclusivement qu'en basse Provence occidentale et en Ardèche méridionale (fig. 14). Dans le détail, les types décoratifs eux-mêmes s'excluent géographiquement : celui des Lauzières est localisé dans 


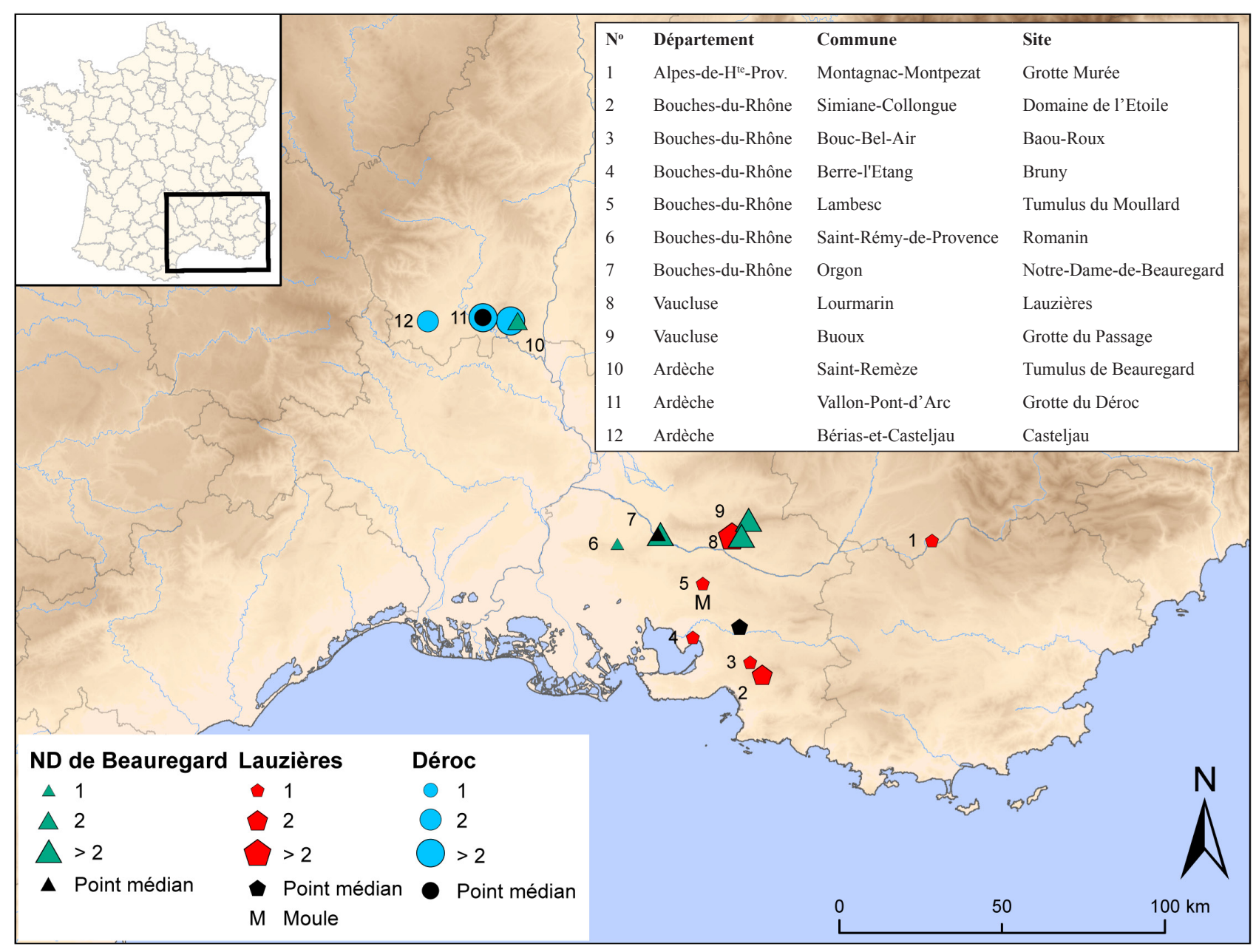

Fig. 14 - Répartition des types décoratifs des bracelets du groupe d'Orgon.

Fig. 14 - Distribution of decorative types of the bracelets of Orgon group.

les zones les plus méridionales, tandis que celui du Déroc est présent dans le Sud de l'Ardèche et celui de NotreDame de Beauregard occupe une position intermédiaire. Les vallées de la Durance et du Rhône font office de zones tampons entre ces différentes aires de répartition. Ces dernières dessinent des espaces de production de ces bracelets, dont la diffusion s'effectuerait principalement à une échelle locale, bien que leurs frontières ne soient pas totalement imperméables. Les points médians de dispersion des types confirment également cette proposition ${ }^{(4)}$. Deux d'entre eux se juxtaposent étonnamment aux deux dépôts ayant donné leurs noms à ces types : NotreDame de Beauregard à Orgon et la grotte du Déroc. Ce résultat vient à nouveau souligner le rôle des contextes de thésaurisation dans les réseaux de diffusion des types métalliques de l'âge du Bronze (Guillemin et Vital, 20062007; Vital et al., 2014).

\section{Indices d'une production locale}

D'autres indices plaident en faveur d'une conception locale de ces parures. Le moule de fondeur du Moullard sud en premier lieu, se trouve dans la zone d'extension du type de bracelets qu'il servait à produire (celui des Lau- zières). Il indique que ces parures étaient coulées dans un moule à valve unique avant d'être cintrées. Dans ce cas, le décor était obtenu directement lors de la fonte. Pour les autres types, l'ornementation du jonc était gravée après ébarbage et polissage de l'objet.

Par ailleurs, ainsi que l'avait déjà fait remarquer A. Müller $(2004)^{(5)}$, les alliages des bracelets du type de Notre-Dame de Beauregard provenant du dépôt éponyme et de l'ensemble 10 des Lauzières présentent des signatures élémentaires semblables (fig. 15), qui laissent penser que ces parures proviennent d'un même atelier, voire que certains objets constituent des « jumeaux de creuset» (Rychner et Kläntschi, 1995). Ils se caractérisent notamment par une proportion élevée de plomb, ce qui concorde avec une datation à une phase récente du Bronze final (Verney et Bocquet, 1998, p.107; Rychner et Stos-Gale, 1998, p. 159-161). Les teneurs en arsenic, nickel et antimoine sont pour leur part considérées comme de bons marqueurs de la matière première utilisée (Dupouy, 1998). Les bracelets du groupe d'Orgon analysés présentent un schéma $\mathrm{Sb}>\mathrm{Ni}>\mathrm{As}$ à teneurs normales (fig. 16), qui les rapproche d'autres objets de la fin du Bronze final des Alpes méridionales, provenant des dépôts de Moriez 
A

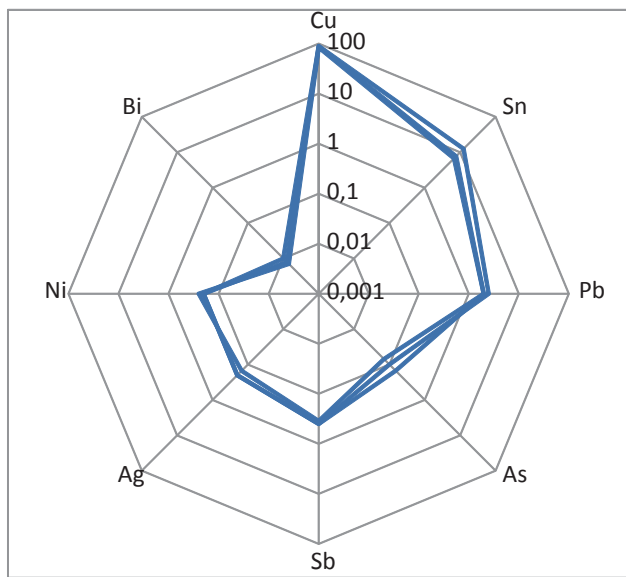

Les Lauzières

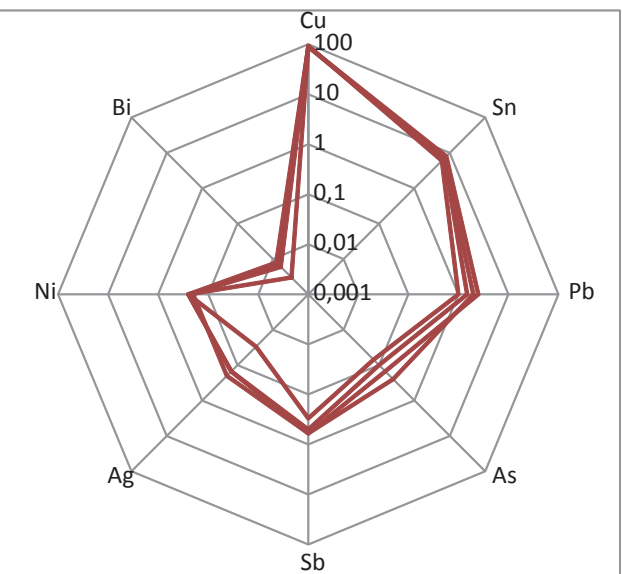

Notre-Dame de Beauregard

B

\begin{tabular}{|l|l|c|c|c|c|c|c|c|c|}
\hline Site & Ill. & $\mathbf{C u}$ & $\mathbf{S n}$ & $\mathbf{P b}$ & $\mathbf{A s}$ & $\mathbf{S b}$ & $\mathbf{A g}$ & $\mathbf{N i}$ & $\mathbf{B i}$ \\
\hline Lourmarin, Les Lauzières & n.d. & 88,8 & 7,7 & 2 & 0,15 & 0,4 & 0,2 & 0,2 & 0,01 \\
\hline Lourmarin, Les Lauzières & n.d. & 84,2 & 6,7 & 2 & 0,07 & 0,35 & 0,15 & 0,25 & 0,007 \\
\hline Lourmarin, Les Lauzières & n.d. & 89,5 & 12,5 & 2,5 & 0,1 & 0,4 & 0,2 & 0,2 & 0,008 \\
\hline Orgon, Notre-Dame de Beauregard & fig. $5, \mathrm{n}^{\circ} 8$ & 90,5 & 7 & 1,5 & 0,1 & 0,5 & 0,15 & 0,25 & 0,006 \\
\hline Orgon, Notre-Dame de Beauregard & fig. $5, \mathrm{n}^{\circ} 9$ & 89,5 & 7 & 2 & 0,25 & 0,6 & 0,15 & 0,25 & 0,008 \\
\hline Orgon, Notre-Dame de Beauregard & fig. $5, \mathrm{n}^{\circ} 7$ & 92,5 & 6 & 1 & 0,07 & 0,3 & 0,03 & 0,25 & 0,003 \\
\hline Orgon, Notre-Dame de Beauregard & fig. $5, \mathrm{n}^{\circ} 10$ & 88,5 & 8 & 2,5 & 0,15 & 0,6 & 0,2 & 0,2 & 0,007 \\
\hline
\end{tabular}

Fig. 15 - A : diagrammes radars de la composition élémentaire des bracelets (type de Notre-Dame de Beauregard) de la nécropole des Lauzières et du dépôt de Notre-Dame de Beauregard. B : détails des résultats des analyses de la composition élémentaires de ces objets (d'après Müller, 2004, corrigé).

Fig. 15 - A: radar diagrams of the elemental composition of the bracelets (Notre-Dame de Beauregard type) from the Lauzières necropolis and from the hoard of Notre-Dame de Beauregard. B: results of the analysis of elemental composition of these objects (after Müller, 2004, corrected).

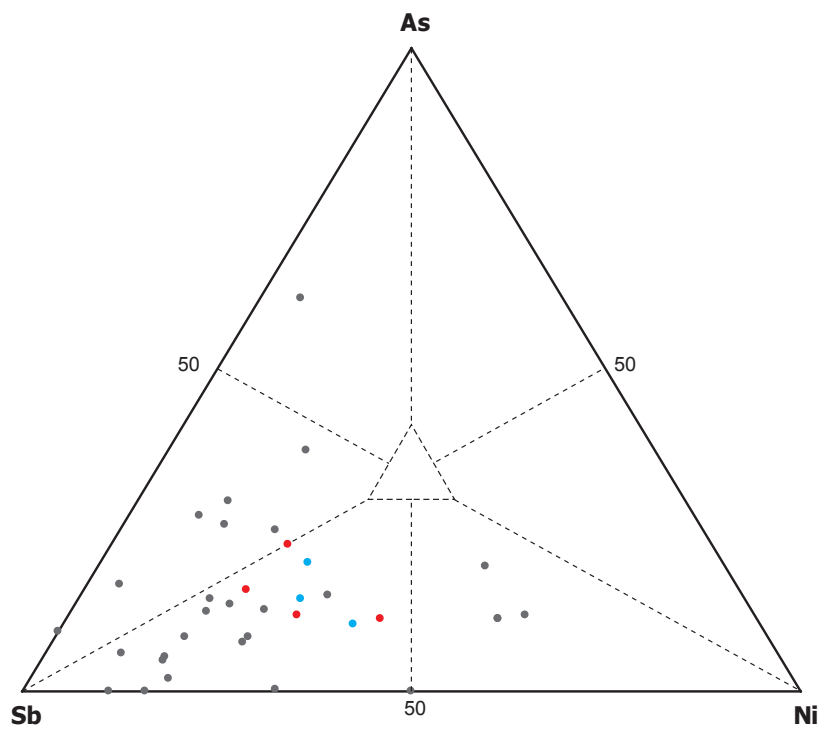

Notre-Dame de Beauregard

Lauzières

bronzes des Alpes méridionales

Fig. 16 - Diagramme ternaire de la teneur en arsenic, nickel et antimoine des bronzes de la nécropole des Lauzières, du dépôt de NotreDame de Beauregard et d'autres sites des Alpes méridionales.

Fig. 16 - Ternary diagram of the content of arsenic, nickel and antimony in the bronzes of the Lauzières necropolis, the hoard of NotreDame de Beauregard and from other sites of the Southern Alps. 
(Barge, 2004), de la Loubière à Saint-Bonnet-enChampsaur, de Saint-Véran et de Saint-Génis (Barge et al., 1998). Ce schéma correspond au type c4 de V. Rychner, qui se retrouve essentiellement au Bronze final 3a (Ha B1) en Suisse (Rychner et Kläntschi, 1995 ; Rychner et Stos-Gale 1998, p. 157), mais qui est majoritaire au Bronze final 3b dans les Alpes du Nord françaises (Verney et Bocquet, 1998, p. 108). Il fait partie des types de composition que P. Northover considère d'origine alpine (Northover, 1982; Rychner et Stos-Gale 1998, p. 158). Il est donc possible que la matière première utilisée pour la confection des bracelets de même type provenant du dépôt d'Orgon et de la nécropole des Lauzières soit issue des minéralisations des Alpes du Sud.

Le groupe d'Orgon n'est en revanche pas représenté dans les dépôts et sépultures de la fin de l'âge du Bronze des départements alpins du Sud-Est de la France. On y retrouve des bracelets beaucoup plus imposants, à tige concave et extrémités en oreillettes dans la sépulture du Grand Ségriès à Moustiers-Sainte-Marie (Courtin et al., 1991) et dans le dépôt de Saint-André-les-Alpes (Ollivier, 1884) ou encore à jonc massif circulaire et côtes transversales dans celui de la Loubière à SaintBonnet-en-Champsaur (Courtois, 1960). Ils participent de parures féminines surabondantes concernant toutes les parties du corps et constituant un poids important de métal (Verger et al., 2007). La création du groupe d'Orgon pourrait répondre à la volonté de produire des parures annulaires moins massives, et donc moins consommatrices en métal, dans une zone plus éloignée des ressources cuprifères.

\section{PARURES ET TERRITOIRES}

工 a majeure partie des exemplaires de chaque type se concentre dans un cercle ne dépassant pas les $50 \mathrm{~km}$ de diamètre. Seulement un ou deux exemplaires par type se trouvent au-delà, à une distance n'excédant pas $100 \mathrm{~km}$ des autres. Ces valeurs s'inscrivent dans l'ordre de grandeur de diffusions des productions locales contraintes par des modes de déplacements traditionnels (à pied, charrette, pirogue), tel qu'observés par A. Gallay dans la boucle intérieure du Niger (Gallay, 2007). Si cette dernière étude concernait le commerce des céramiques, les différents modes d'acquisitions observés (déplacements des artisans, des acheteurs ou fréquentations des marchés) sont tout à fait semblables à ceux que l'on peut attendre pour les industries métalliques protohistoriques.

Pour expliquer cette association d'un type décoratif à un territoire donné, deux hypothèses principales s'offrent à nous. La première est celle de l'existence de trois ateliers spécialisés possédant chacun un type d'ornementation spécifique, sorte de marque de fabrique. Les indices de productions locales présentés précédemment ne contredisent pas cette hypothèse, de même que la coïncidence des centres médians de dispersion avec les contextes de dépôts. Néanmoins, les différents exemplaires d'un même type présentent eux-mêmes une variabilité dans l'exécution des motifs, plus compatible avec l'existence de plusieurs artisans. Une seconde hypothèse est que ces décors soient les supports d'expressions identitaires. Leur zone de répartition principale pourrait ainsi correspondre à un territoire communautaire, tandis que les quelques exemplaires plus éloignés seraient les reflets de pratiques d'échanges. Les distances enregistrées peuvent ainsi correspondre à l'existence de réseaux familiaux tels qu'ils ont été observés au Niger (Gosselain, 2008, fig. 6). De nombreux anthropologues ont d'ailleurs souligné le rôle des objets de parures dans l'affirmation d'identités ethniques ou culturelles (Vanhaeren et D'Errico 2011, p. 11). On sait par exemple que dans l'orfèvrerie berbère, « les modes de décorations des bracelets répondent au style de chaque région et souvent de chaque artisan » (CampsFabrer, 1991, p. 38). Chez les Touareg également, certains signes gravés sur la parure correspondent à des « marques de famille » (Dieterlen et Liegers, 1972, p. 30).

Les données fournies par d'autres manifestations de la culture matérielle de la fin de l'âge du Bronze, la céramique en particulier, confortent les informations territoriales indiquées par la répartition des bracelets du groupe d'Orgon. Leur aire de diffusion s'inscrit principalement en basse Provence occidentale, dont l'homogénéité du faciès céramique au Bronze final $3 b$ a pu être établie par une analyse statistique (Lachenal, 2011, p. 250, fig. 15). Néanmoins, dans le détail, quelques différences stylistiques et décoratives peuvent y être décelées, dépendant des influences diversifiées que connait ce territoire. Ainsi, les décors figuratifs au trait double, caractéristiques du faciès Mailhac I languedocien (Guilaine, 1972), sont surtout localisés dans le bas Rhône, sur les sites de la Calade à Fontvielle, de Saint-Blaise à Saint-Mitre-les-Remparts ou de l'Abion à Martigues (Bouches-du-Rhône). Une incursion vers le Verdon est également documentée par l'abri du Jardin du Capitaine (Alpes-de-Haute-Provence). À l'inverse, les affinités avec les styles de France médiane se retrouvent surtout sur les sites plus septentrionaux, du Vaucluse ou des Alpes-de-Haute-Provence comme la grotte Basse à Cheval-Blanc ou la grotte Murée (Lachenal, 2011, p. 250). Ces différences enregistrées de part et d'autre de la Durance ne sont pas sans évoquer la répartition des bracelets des types des Lauzière et de Notre-Dame de Beauregard. Celui du Déroc se retrouve pour sa part uniquement en Ardèche méridionale où les productions céramiques, proches de celles de la moyenne vallée du Rhône, montrent dès le Bronze final $3 \mathrm{a}$ des liens privilégiés avec les régions septentrionales (Vital, 2014, p. 76).

\section{CONCLUSION}

$M$ arqueurs identitaires ou sociaux, objets d'échanges ou systèmes de communication, les parures constituent des supports d'expressions polysémiques au poten- 
tiel informatif extrêmement fécond (Vanhaeren et D'Errico 2011). À l'âge du Bronze, la variété morphologique et décorative dont fait preuve cette catégorie d'objets en fait depuis longtemps des supports privilégiés dans la définition de groupes culturels, de réseaux d'échanges ou d'horizons chronologiques. Cette étude nous montre qu'un niveau d'observation plus précis peut être atteint. L'analyse détaillée de ce corpus de bracelets du Sud-Est de la France permet en effet d'identifier une production microrégionale propre à la dernière phase de l'âge du Bronze, faisant preuve d'une variabilité décorative à l'échelle locale. Cette dernière peut être mise en relation avec des ateliers et des réseaux d'échanges privilégiés qui se superposent à ceux identifiés par l'intermédiaire des productions céramiques. Elle contribue ainsi à la caractérisation des groupes culturels du bassin inférieur du Rhône à l'extrême fin de l'âge du Bronze. Localisés à distance des ressources en cuivre des Alpes et du sud du Massif central, ils ne sont néanmoins pas dépourvus d'une production métallurgique propre, dont les bracelets du groupe d'Orgon constituent les témoins les plus directs.

Remerciements : Je remercie vivement André D'Anna pour la communication de documents relatifs à la fouille des Lauzières à Lourmarin, Michèle Bois et Amicie d'Arces pour l'accès au bracelet d'" Orange » provenant de la collection Vallentin du Cheylard, Jean Gascó et Gwendoline Borja pour m'avoir fourni leurs dessins des anneaux du Déroc, ainsi que Bernard Dedet et Éric Durand pour les informations et les illustrations concernant la nécropole de Beauregard. Ce travail est inscrit dans l'axe thématique «IDENTITÉS : Contacts de cultures et formes d'acculturation en Méditerranée » du programme scientifique du Labex ARCHIMÈDE, programme IA- ANR-11LABX-0032-01.

\section{NOTES}

(1) Nous utilisons dans cet article la chronologie actualisée pour la Provence à partir de l'analyse conjointe de la sériation des productions céramiques et des datations par le radiocarbone pouvant leur être associées (Lachena, 2014). Dans un souci de continuité, l'architecture du système chronologique proposé par J.-J. Hatt a été conservée, mais celui-ci a été réactualisé et adapté en fonction des données locales. Pour marquer cette différence, les chiffres romains traditionnellement utilisés pour distinguer les subdivisions de périodes ont été remplacés par des chiffres arabes.

(2) Dans les publications de ces bracelets, il semblerait que l'un d'eux ait été dessiné deux fois (D'Anna et al., 2002, fig. $101 \mathrm{n}^{\text {os }} 1$ et 2 ; Müller, 2004, fig. $1 \mathrm{n}^{\text {os }} 1$ et 2).

(3) Numéro d'inventaire 84.85.731(626).

(4) Les points médians de dispersion ont été calculés à l'aide du logiciel ArcGIS10.1.

(5) Une erreur de retranscription de la teneur en nickel d'un objet des Lauzières a pu être corrigée grâce à la consultation du rapport original de J. R. Bourhis.

\section{RÉFÉRENCES BIBLIOGRAPHIQUES}

BARGE H. (2004) - Le dépôt de bronzes de Moriez (Alpes-deHaute-Provence), Documents d'archéologie méridionale, 27, p. 141-170.

Barge H., Bourhis J.-R., Rostan P. (1998) - Métallurgie préhistorique et gîtes cuprifères dans le Sud-Est de la France : premiers résultats, in A. D'Anna et D. Binder (dir.), Production et identité culturelle, Actualité de la recherche, actes des rencontres méridionales de Préhistoire récente (Arles, 8-9 novembre 1996), Antibes, Éditions APDCA, p. 65-80.

Benfoughal T. (1998) - Bijoux et bijoutiers de l'Aurès. Traditions et innovations, Paris, CNRS, $252 \mathrm{p}$.

Boissinot P. (1993) - Archéologie de l'habitat protohistorique. Quelques points méthodologiques (historiographie et épistémologie) examinés à partir de la fouille d'une agglomération de la périphérie massaliète, thèse de doctorat, École des hautes études en sciences sociales, Toulouse, $738 \mathrm{p}$.

Boissinot P., Cordier L., Marrou P. (1998) - Un transect dans le vallon du Bourrely à Lambesc, Bouches-du-Rhône (opération TGV Méditerranée). Morphogénèse et habitat préhistorique, in A. D'Anna et D. Binder (dir.), Production et identité culturelle, Actualité de la recherche, actes des rencontres méridionales de Préhistoire récente (Arles, 8-9 novembre 1996), Antibes, APDCA, p. 393-401.

CAmpolo S., Garcia D. (2004) - Bronzes protohistoriques $d u$ musée Calvet d'Avignon, Avignon, musée Calvet, 120 p.
CAmps-Fabrer H. (1991) - Bijoux, in G. Camps (dir.), Encyclopédie berbère, 10. Beni Isguen - Bouzeis, Aix-en-Provence, Édisud, p. 1496-1516.

CongÈs G. (1982) - Le gisement du col Sainte-Anne à SimianeCollongue (Bouches-du-Rhône). Le matériel de l'âge du Bronze, Bulletin archéologique de Provence, 9, p. 13-18.

Cordier L. (2002) - Occupation du Bronze final IIIb dans un fond de vallon au Moullard (Lambesc), in Archéologie du TGV Méditerranée, Fiches de synthèse, 2. La Protohistoire, Lattes, ARALO (Monographies d'archéologie méditerranéenne, 9), p. 561-566.

Cordier G. (2009) - L'Âge du Bronze dans les Pays de la Loire moyenne, Joué-lès-Tours, La Simarre, 702 p.

Cotte J. (1924) - Étude des ossements humains de la station néolithique de Bruny sur l'étang de Berre, Revue anthropologique, 34, p. 283-294.

Courtin J., Legros T., Muller A. (1991) - La sépulture du Grand Ségriès à Moustiers-Sainte-Marie (Alpes-deHaute-Provence), in A. Duval (dir.), Les Alpes à l'âge du Fer, Paris, CNRS (Revue archéologique de Narbonnaise, suppl. 22), p. 283-288.

Courtors J.-C. (1957) - Le dépôt de fondeur de « la Farigourière » à Pourrières (Var), Cahiers rhodaniens, 4, p. 36-48.

Courtois J.-C. (1960) - L'âge du Bronze dans les HautesAlpes, Gallia préhistoire, 3, p. 47-108. 
D’anna A., Muller A., Coutel R., Courtin J. (2002) - Les Lauzières (Lourmarin), in J. Buisson-Catil et J. Vital (dir.), Âges du Bronze en Vaucluse, Avignon, A. Barthélemy et département de Vaucluse (Notices d'archéologie vauclusienne, 5; Travaux du Centre d'archéologie préhistorique de Valence, 4), p. 215-225.

David-Elbiali M., Dunning C. (2005) - Le cadre chronologique relatif et absolu au nord-ouest des Alpes entre 1060 et 600 av. J.-C., in G. Bartoloni et F. Delpino (dir.) - Oriente e Occidente : metodi e discipline a confronto. Riflessioni sulla cronologia dell'età del Ferro italiana, actes de la rencontre d'étude (Rome, 30-31 octobre 2003), Pise - Rome, Istituto Editoriali e Poligrafici internazionali (Mediterranea, 1), p. 145-195.

DedeT B. (2001) - Tombes et pratiques funéraires protohistoriques des Grands Causses du Gévaudan (Aveyron, Gard, Lozère), Paris, MSH (Documents d'archéologie française, 84), $356 \mathrm{p}$.

Dedet B. (2002) - Archéologie funéraire protohistorique sur le tracé du TGV dans la vallée du Rhône et en Provence occidentale, in Archéologie du TGV Méditerranée, Fiches de synthèse, 2. La Protohistoire, Lattes, ARALO (Monographies d'archéologie méditerranéenne, 9), p. 351-358.

Dedet B., Bordreuil M. (1982) - Le dépôt de fondeur du Bronze final II de Cabanelle à Castelnau-Valence (Gard), Gallia Préhistoire, 25, 1, p. 187-210.

Dedet B., Durand E. (2016) - Sépultures et pratiques funéraires en Ardèche méridionale au Bronze final III et au premier âge du Fer, Documents d'archéologie méridionale, 38, p. 33-128.

Dieterlen G., Ligers Z. (1972) - Contribution à l'étude des bijoux touareg, Journal de la société des africanistes, 42, 1, p. $29-53$

Dupouy J.-M. (1998) - Réflexions sur l'intérêt des proportions relatives des teneurs en impuretés métalliques et de l'analyse des impuretés non-métalliques pour la connaissance de la fabrication des Bronzes, in C. Mordant, M. Pernot et V. Rychner (dir.), L'Atelier du bronzier en Europe du XX $X^{e}$ au VIII ${ }^{e}$ siècle avant notre ère, 1. Les analyses de composition du métal : leur apport à l'archéologique de l'âge du Bronze, actes du colloque international «Bronze 96 » (Neuchâtel et Dijon, 6-9 mars 1996), Paris, CTHS, p. 41-52.

Èluère C., Gomez J. (1990) - Typologie des objets de l'âge du Bronze en France, fascicule VII. Colliers, bracelets, boucles, Paris, Société préhistorique française, $163 \mathrm{p}$.

Gachina J., Gomez De Soto J., Bourhis J.-R., Veber C. (2008) - Un dépôt de la fin de l'âge du Bronze à Meschers (Charente-Maritime). Remarques sur les bracelets et tintinnabula du type de Vaudrevange en France de l'Ouest, Bulletin de la Société préhistorique française, 105, 1, p. 159-185.

Gallay A. (2007) - Les jarres de mariage décorées du delta intérieur du Niger (Mali) : Essai de délimitation archéologique, The Arkeotek Journal, 1, 1, http://www.thearkeotekjournal.org/tdm/Arkeotek/fr/archives/2007/1Gallay.xml [en ligne].

Gascó J., BorJa G. (2016) - Le dépôt de bronzes de la grotte du Déroc à Vallon-Pont-d'Arc (Ardèche) : révision et nouveautés, Documents d'archéologie méridionale, 37, p. 51-71.
Gosselain O. P. (2008) - Thoughts and Adjustments in the Potter's Backyard, in I. Berg (dir.), Breaking the Mould: Challenging the Past through Pottery, Oxford, Archaeopress (BAR, International Series 1861), p. 67-79.

Guilaine J. (1972) - L'Âge du bronze en Languedoc occidental, Roussillon, Ariège, Paris, Kliencksieck (Mémoire de la Société préhistorique française, 9), $460 \mathrm{p}$.

Guillemin H., Vital J. (2006-2007) - La hache de Marignane (Bouches-du-Rhône) et la typologie des haches du type de Boismurie à l'âge du Bronze moyen, Documents d'archéologie méridionale, 29-30, p. 7-17.

Haussmann L. (1996-1997) - L'âge du Bronze dans les Hautes-Alpes, Bulletin d'études préhistoriques et archéologiques alpines, 7-8, p. 97-180.

JANIN T. (1992) - L'évolution du Bronze final IIIb et la transition Bronze-Fer en Languedoc occidental d'après la culture matérielle et les nécropoles, Documents d'archéologie méridionale, 15, p. 243-259.

Lachenal T. (2009) - De l'âge du Bronze à l'âge du Fer dans les gorges du Verdon (Var, Alpes-de-Haute-Provence) : analyse critique des ensembles stratigraphiques, in M.-J. Roulière-Lambert, A. Daubigney, P.-Y. Milcent, M. Talon et J. Vital (dir.), De l'âge du Bronze à l'âge du Fer en France et en Europe occidentale ( $X^{e}$-VII siècle av. J.-C.). La moyenne vallée du Rhône aux âges du Fer, actes du $\mathrm{XXX}^{\mathrm{e}}$ Colloque international de l'AFEAF, co-organisé avec l'APRAB (Saint-Romain-en-Gal, 2006), Dijon, RAE (Revue archéologique de l'Est, supplément 27), p. 527-534.

Lachenal T. (2010) - L'âge du Bronze en Provence : productions céramiques et dynamiques culturelles, thèse de doctorat, université de Provence, Aix-en-Provence, 446 p.

Lachenal T. (2011) - Entre Alpes et Méditerranée. Productions céramiques et dynamiques culturelles de la fin de l'âge du Bronze en Provence ( $\mathrm{X}^{\mathrm{e}}$-IX $\mathrm{X}^{\mathrm{e}}$ s. av. J.-C.), Quaderns de Prehistòria i Arqueologia de Castelló, 29 (La edad del bronce en el Mediterráneo occidental), p. 231-267.

Lachenal T. (2014) - Chronologie de l'âge du Bronze en Provence, in I. Sénépart, F. Léandri, J. Cauliez, T. Perrin et É. Thirault (dir.), Chronologie de la Préhistoire récente dans le Sud de la France. Acquis 1992-2012. Actualité de la recherche, actes des $\mathrm{X}^{\mathrm{es}}$ Rencontres méridionales de Préhistoire récente (Porticcio, 18-20 octobre 2012), Toulouse, Archives d'écologie préhistorique, p. 197-220.

Lagrand C.-H. (1968) - Recherches sur le Bronze final en Provence méridionale, thèse de doctorat, université d'Aix-enProvence, $394 \mathrm{p}$.

Lagrand C. (1987) - Le premier âge du Fer dans le Sud-Est de la France, in F. Fischer (dir.), Hallstatt-Studien = Études hallstattiennes, Tübingen, Acta Humanoria (Quellen und Forschungen zur prähistorischen und provincialrïmischen Archäologie, 2), p. 44-55

Milcent P.-Y. (1998) - Le Petit-Villatte à Neuvy-sur-Barangeon (Cher) : lecture d'un dépôt complexe, in C. Mordant, M. Pernot et V. Rychner (dir.), L'Atelier du bronzier en Europe du XXe au VIII siècle avant notre ère, III. Production, circulation et consommation du bronze, actes du colloque international « Bronze 96 » (Neuchâtel et Dijon, 6-9 mars 1996), Paris, CTHS, p. 55-70. 
Milcent P.-Y. (2009) - À l'Est rien de nouveau. Chronologie des armes de poing du premier âge du Fer médio-atlantique et genèse des standards matériels élitaires hallstattiens et laténiens, in A. Lehoërff(dir.), Construire le temps. Histoire et méthodes des chronologies et calendriers des derniers millénaires avant notre ère en Europe occidentale, actes $\mathrm{du} \mathrm{XXX}^{\mathrm{e}}$ Colloque international HALMA-IPEL (Lille, 7-9 décembre 2006), Glux-en-Glenne, Centre archéologique européen (Bibracte, 16), p. 231-250.

MüLler A. (2004) - Le mobilier métallique de la nécropole des Lauzières (Lourmarin, Vaucluse) et celui du dépôt de bronzier de Notre-Dame de Beauregard (Orgon, Bouchesdu-Rhône), in J. Buisson-Catil, A. Guilcher, C. Hussy, M. Olive et M. Pagni (dir.), Vaucluse préhistorique : le territoire, les hommes, les cultures et les sites, Le Pontet, Barthélémy, p. 290-293.

Northover P. (1982) - The Exploration of the Long-distance Movement of Bronze in Bronze and Early Iron Age Europe, Bulletin of the Institute of Archaeology, University of London, 19, p. 45-72.

Ollivier A. (1884) - Âge préhistorique dans les Basses-Alpes. Période du Bronze, Annales des Basses-Alpes. Bulletin de la Société scientifique et littéraire des Basses-Alpes, 13, p. 189-192.

Ricalens H., Soutou A. (1965) - Les bracelets de bronze de Carlipa (Aude), Gallia Préhistoire, 8, p. 13-20.

Rolland H. (1938) - Ossuaire de Romanin (Saint-Rémyde-Provence), Bulletin de la Société préhistorique française, 35,12 , p. 470-479.

Roudil J.-L. (1990) - Le trésor du Déroc, Ardèche archéologie, 7, p. 29-33.

Rychner V., Klantschi N. (1995) - Arsenic, nickel et antimoine. Une approche de la métallurgie du Bronze moyen et final en Suisse par l'analyse spectrométrique, Lausanne, CAR (Cahiers d'archéologie romande, 63), 112 p.

Rychner V., Stos-Gale Z. (1998) - Compositions chimiques et isotopes du Plomb : la production métallique de l'âge du Bronze moyen et final en Suisse, in C. Mordant, M. Pernot et V. Rychner (dir.), L'Atelier du bronzier en Europe $d u X X^{e}$ au VIII ${ }^{e}$ siècle avant notre ère, I. Les analyses de composition du métal : leur apport à l'archéologique de l'âge du Bronze, actes du colloque international «Bronze 96 » (Neuchâtel et Dijon, 6-9 mars 1996), Paris, CTHS, p. 153-174.

TAfFanel O., TAFfanel J., Janin T. (1998) - La nécropole $d u$ Moulin à Mailhac (Aude), Lattes, ARALO (Monographies d'archéologie méditerranéenne, 2), 393 p.

Vanhaeren M., D'Errico F. (2011) - L'émergence du corps paré, Civilisations, 59, 2, p. 59-86.

Verger S. (1992) - L'épée du guerrier et le stock de métal : de la fin du Bronze ancien à l'âge du Fer, in P. Curdy et G. Kaenel (dir.), L'âge du fer dans le Jura, actes du XVe Colloque de l'Association française pour l'étude de l'âge du Fer (Pontarlier et Yverdon-les-Bains, 9-12 mai 1991), Lausanne, Bibliothèque historique vaudoise et Lons-le-Saunier, Cercle Girardot, p. 135-151.
Verger S. (1998) - Les trois âges de la dame de Blanot, in C. Mordant, M. Pernot et V. Rychner (dir.), L'Atelier du bronzier en Europe du XX $X^{e}$ au VIII siècle avant notre ère, III. Production, circulation et consommation du bronze, actes du colloque international « Bronze 96 » (Neuchâtel et Dijon, 6-9 mars 1996), Paris, CTHS, p. 33-39.

Verger S., Dumont A., Moyat P., Mille B., (2007) - Le dépôt de bronzes du site fluvial de la Motte à Agde (Hérault), Jahrbuch des Römisch-Germanischen Zentralmuseums Mainz, 54, p. 85-171.

Verney A., BocQuet A. (1998) - La composition élémentaire d'objets du Bronze final des Alpes françaises du Nord : premiers résultats, in $\mathrm{C}$. Mordant, $\mathrm{M}$. Pernot et $\mathrm{V}$. Rychner (dir.), L'Atelier du bronzier en Europe du XX ${ }^{e}$ au VIII ${ }^{e}$ siècle avant notre ère, I. Les analyses de composition du métal : leur apport à l'archéologique de l'âge du Bronze, actes du colloque international « Bronze 96 » (Neuchâtel et Dijon, 6-9 mars 1996), Paris, CTHS, p. 101-118.

Vital J. (1999) - Identification du Bronze moyen-récent en Provence et en Méditerranée nord-occidentale, Documents d'archéologie méridionale, 22, p. 7-115.

Vital J. (2002a) - Les sites du vallon de l'Aiguebrun (Buoux), in J. Buisson-Catil et J. Vital (dir.), Ages du Bronze en Vaucluse, Avignon, A. Barthélemy et département de Vaucluse (Notices d'archéologie vauclusienne, 5; Travaux du Centre d'archéologie préhistorique de Valence, 4), p. 84-93.

Vital J. (2002b) - Les sites de Buoux (Buoux), in J. BuissonCatil et J. Vital (dir.), Âges du Bronze en Vaucluse, Avignon, A. Barthélemy et département de Vaucluse (Notices d'archéologie vauclusienne, 5; Travaux du Centre d'archéologie préhistorique de Valence, 4), p. 212-213.

Vital J. (2002c) - Grotte des Epingles (Cheval-Blanc), in J. Buisson-Catil et J. Vital (dir.), Ages du Bronze en Vaucluse, Avignon, A. Barthélemy et département de Vaucluse (Notices d'archéologie vauclusienne, 5; Travaux du Centre d'archéologie préhistorique de Valence, 4), p. 127-134.

Vital J. (2014) - Repères chronométriques, typologiques et géographiques pour la céramique du Bronze final du Rhône aux Alpes, Documents d'archéologie méridionale, 35, p. 53-83.

Vital J., Cattin F., Letterlè F., Degrypse P., (2014) - Un dépôt d'épées courtes du Bronze ancien : la Rouvière à Chusclan (Gard), Bulletin de la Société préhistorique française, 111, 1, p. 75-100.

Thibault LACHENAL chargé de recherche CNRS

UMR5140 Archéologie des sociétés méditerranéennes (ASM), univ. Paul-Valéry Montpellier, CNRS, MCC, F-34000 Montpellier, France thibault.lachenal@cnrs.fr 